\title{
Collimation method studies for next-generation hadron colliders
}

\author{
Jian-Quan Yang, ${ }^{1,2}$ Ye Zou, ${ }^{3}$ and Jing-Yu Tang ${ }^{1,2,4, *}$ \\ ${ }^{1}$ Institute of High Energy Physics, CAS, Yuquan Road 19B, Beijing 100049, People's Republic of China \\ ${ }^{2}$ University of Chinese Academy of Sciences, CAS, Yuquan Road 19A, Beijing 100049, \\ People's Republic of China \\ ${ }^{3}$ Uppsala University, Uppsala 75120, Sweden \\ ${ }^{4}$ Dongguan Neutron Science Center, Dongguan 523803, People's Republic of China
}

(Received 2 July 2018; published 19 February 2019)

\begin{abstract}
In order to handle the extremely high stored energy in future proton-proton colliders, an extremely highefficiency collimation system is required for safe operation. At the LHC, the major limiting locations in terms of particle losses on superconducting (SC) magnets are the dispersion suppressors downstream of the transverse collimation insertion. These losses are due to the protons experiencing single diffractive interactions in the primary collimators. How to solve this problem is very important for future protonproton colliders, such as the Future Circular Hadron-Hadron Collider and the Super Proton-Proton Collider. In this article, a novel method is proposed, which arranges both the transverse and momentum collimation in the same long straight section. In this way, additional absorbers between the two cleaning hierarchies can clean those particles related to the single diffractive effect, with the downstream momentum collimation system intercepting any further leakage. The effectiveness of the method has been confirmed by multiparticle simulations. In addition, SC quadrupoles with special designs such as an enlarged aperture and good shielding are adopted to enhance the phase advance in the transverse collimation section so that tertiary collimators can be arranged to clean off the tertiary halo which emerges from the secondary collimators and improve the collimation efficiency. With one more collimation stage in the transverse collimation, the beam losses in both the momentum collimation section and the experimental regions can be largely reduced. Multiparticle simulation results with the MERLIN code confirm the effectiveness of the collimation method. At last, we provide a protection scheme of the SC magnets in the collimation section. The FLUKA simulations show that, by adding some special protective collimators in front of the magnets, the maximum power deposition in the SC coils is reduced dramatically, which is proven to be valid for protecting the SC magnets from quenching.
\end{abstract}

DOI: 10.1103/PhysRevAccelBeams.22.023002

\section{INTRODUCTION}

For high-energy proton-proton colliders, superconducting (SC) magnets are essential to achieve the magnetic strength required to reach a higher center of mass energy. These magnets have an increasing sensibility to particle losses, which scales in level with the magnetic strength. A tiny fractional loss of the full beam in SC magnet coils, even the radiation from the particles lost in other locations, could lead to a quench; thus, any significant beam loss in the SC magnets (also called cold magnets with respect to warm magnets for room-temperature magnets) must be

\footnotetext{
*Corresponding author. tangjy@ihep.ac.cn

Published by the American Physical Society under the terms of the Creative Commons Attribution 4.0 International license. Further distribution of this work must maintain attribution to the author(s) and the published article's title, journal citation, and DOI.
}

avoided. Large beam losses could also cause serious damage to other accelerator components. However, beam losses cannot be completely suppressed because of various beam dynamic processes, such as beam-beam interactions, transverse and longitudinal diffusion, residual gas scattering, and so on [1]. Therefore, besides strictly controlling beam losses and a very reliable beam abort system, a robust and extremely efficient collimation system is necessary to safely dispose of the beam losses. At the LHC [2], the highest-energy collider in the world nowadays, a very complex collimation system with the multistage collimation method was designed, and a total of 132 pure collimators were designed, including primary collimators, secondary collimators, absorbers, tertiary collimators, and other protection collimators, which have been and will be installed in a phased approach [3-5]. During the LHC run I, the beam energy was up to $4 \mathrm{TeV}$ and the stored beam energy was up to $143 \mathrm{MJ}$, the collimation system with the so-called tight settings [6] accomplished its tasks very well [7-8], a record cleaning inefficiency below a few $10^{-4}$ was 
achieved in the cold regions where they were filled with SC magnets, and the strictest control of beam losses was required. As to the LHC run II, the beam energy was increased from 4 to $6.5 \mathrm{TeV}$, and a shorter bunch spacing of $25 \mathrm{~ns}$ was adopted. With a higher stored energy of about $270 \mathrm{MJ}$, the performance of the collimation system is still very good [9-10]. However, when the LHC is upgraded to the High-Luminosity Large Hadron Collider (HL-LHC) with higher stored energy, more particle losses in the dispersion suppressors (DSs) downstream of the transverse collimation insertion is considered threatening to the local cold dipoles $[8,11]$. These losses are due to the protons experiencing single diffractive interactions in the primary collimators [12]. Such protons can survive the interactions and emerge from the collimator jaws with their momentum modified only slightly in direction but significantly in magnitude. In other words, this process converts the transverse halo particles into off-momentum halo particles. Thus, those protons will be able to escape from the transverse collimation system and are lost as soon as they reach the downstream DS section. In order to largely reduce the irradiation dose rate to the SC magnets at the downstream DS section of the betatron collimation insertion (IR7) for each beam, two local collimators need to be added in the DS section. However, there is not enough space for additional collimators due to the compact design in the DS region. One solution to create space for additional collimators is to move the cold magnets in the DS section [13]. Another solution is to replace the two original main dipoles of $8.3 \mathrm{~T}$ by two new shorter dipoles with new $\mathrm{Nb}_{3} \mathrm{Sn}$ magnet technology which can work at $11 \mathrm{~T}$ [14].

For the design of future proton-proton colliders, due to the increasing probability of single diffractive interaction with the increase in energy [15-18], the problem of beam losses in the DSs where the dispersion starts to increase becomes more important and should be treated with greater care. For the Future Circular Hadron-Hadron Collider (FCC-hh), an analogous solution to the HL-LHC with local dedicated protection collimators in the DSs is being studied [19]. In this paper, a different approach is presented, which arranges both the transverse and momentum collimation in the same cleaning insertion. In this way, the downstream momentum collimation system that includes the protective absorbers at dispersive locations and a multistage momentum collimation system will clean off the particles with a large momentum deviation including those that experience single diffractive interactions with the primary transverse collimators. Then one can get rid of beam losses in the DS regions and design the arc lattice as compact as possible. However, the challenge of this method is how to join two different collimation sections. In general, the transverse collimation section is designed to be approximately dispersion-free, but relatively large dispersions are required at the locations of primary collimators in the momentum collimation section [20]. Different from
Dipole

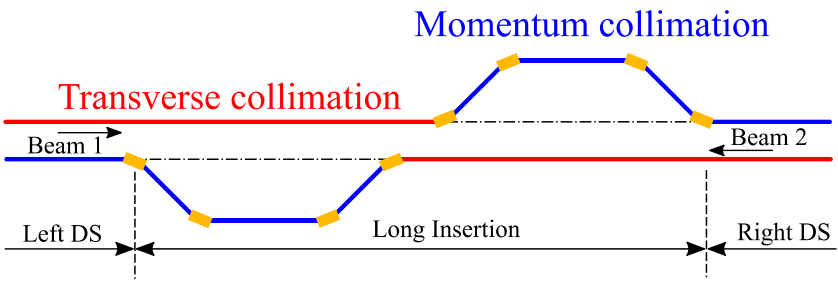

FIG. 1. Layout of the combined transverse and momentum collimation method.

the momentum collimation section at the LHC, where dispersion is intentionally designed to be nonzero between the two adjacent DS sections, thus a chicanelike and achromatic design for the momentum collimation section is adopted here. The schematic of the method is depicted in Fig. 1, where arc dipole magnets with a simplified design of only a single aperture instead of a twin aperture are used, where the associated cryomodules should be designed specially to allow the pass-through of another beam pipe. In this way, sufficient longitudinal space can be available here to add necessary protective collimators and shielding in room temperature. Detailed studies including lattice design and multiparticle simulations using the MERLIN code $[18,21-23]$ have been carried out to check the validity of the method. The result shows that this method works as expected and the beam losses at the downstream DSs can be suppressed. In addition, SC quadrupole magnets with special protection are being considered to provide more phase advance in the transverse collimation section, where room-temperature magnets are used due to the high radiation dose rate. This measure can enhance the transverse collimation efficiency.

For a next-generation hadron collider, the above method is a general and applicable solution for the collimation system. As the first conceptual approach phase, the studies presented here are mainly based on the parameters of the Super Proton-Proton Collider (SPPC), which is the second phase of the CEPC-SPPC project [24]. The layout and main parameters of the SPPC are given in the Appendix.

\section{LATTICE FOR THE COMBINED BETATRON AND MOMENTUM COLLIMATION}

The top beam kinetic energy of the SPPC in the baseline design is $37.5 \mathrm{TeV}$, which is about 5 times that at the LHC. The energy of halo particles is too high to be dissipated in a straightforward way. One general method to stop highenergy protons is to use multistage collimators. Depending on the collimators' functions, they are divided into several families. The primary collimators will intercept or scatter the primary halo particles, and the secondary collimators will intercept the secondary beam halos that are formed by the particle's interaction with the primary collimators. Sometimes, tertiary collimators will intercept the so-called 
tertiary beam halos (i.e., what emerges from the secondary collimators). At the LHC, tertiary collimators are placed at the interaction points (IPs), where they protect the bottlenecks, represented by the inner triplets at the IPs with the smallest $\beta^{*}$ (the beta function at the interaction point). The absorbers will stop the hadron showers from the upstream collimators, and additional collimators are used to protect the SC magnets.

As critical components in ensuring the safe operation of an accelerator like the SPPC, based on the experience at the LHC [19], the material of collimators would have to meet requirements: good conductivity to reduce coupling impedance, high robustness to resist abnormal beam impacts, and good absorption ability for cleaning efficiency [4]. Unfortunately, not all three conditions can be fulfilled by the same material. A robust material, such as graphite, would increase the coupling impedance, which is important for collective beam instabilities and limits the machine performance. On the other hand, a good conductor, such as copper, is not robust enough, which means that the collimator jaws can be damaged even in the normal operation mode. Thus, different materials will be used. As the closest objects to the circulating beam, the primary and secondary collimators must withstand the highest dose of deposited energy without permanent damage. For this reason, they are made of a robust carbon fiber-carbon composite. However, referring to the collimation study for the FCC-hh [25-26], for an extremely high stored beam energy of about $10 \mathrm{GJ}$, assuming the total beam loss within $0.2 \mathrm{hr}$ in the transverse collimation section, the primary or secondary collimators have to resist a power load of several hundreds of kilowatts, which is extremely challenging for the robustness of the collimators. As for the tertiary collimators and absorbers, due to a lower heat power load, they are made of a high- $Z$ material, such as copper and tungsten, which can absorb particles efficiently and reduce the impedance relatively. Meanwhile, the number of collimators and sharing of phase space coverage can ensure that the large level of energy deposition is distributed among them, avoiding a single device being overloaded. Thus, the location for each collimator needs to be optimized according to the $\beta$ functions, in order to obtain larger gap openings to reduce impedance issues and obtain an appropriate phase advance between collimators to improve the cleaning efficiency.

\section{A. Requirements for the lattice design for transverse collimation}

At the LHC, collimators represent more than $90 \%$ of the impedance of all the accelerator components [27], and they produce the transverse wall impedance which scales inversely proportional to the third power of the collimator gap size. Thus, one effective method to reduce the impedance is to enlarge the collimator gap, which means that the collimators must be located at large $\beta$ values in the case of the unchanged ratio of gaps over a beam size in $\sigma$ (normalized transverse rms beam radius). In addition, with a larger $\beta$, the same change in the Courant-Snyder invariant means a larger change in the amplitude, which enhances the impact parameter and reduces the outscattering probability. Therefore, the $\beta$ function is required to be larger in the collimation insertion than in the arcs. To have high collimation efficiency in a multistage collimation system, the phase advance between different stages of collimators is also very important; thus, a long insertion is needed to produce enough phase advances.

For proton accelerators, the transverse collimation plays a major role relative to the momentum collimation; thus, the former has higher requirements for the lattice design and collimators and will withstand higher radiation doses. According to the principles of two-stage betatron collimation [28], in the one-dimensional case, the optimal phase advance $\mu_{\mathrm{opt}}$ should satisfy

$$
\cos \mu_{\mathrm{opt}}= \pm n_{1} / n_{2}
$$

where $n_{1}$ and $n_{2}$ donate the apertures of primary and secondary collimators in units of $\sigma$, respectively. For the two-dimensional case, it becomes complicated. At the LHC, the long straight sections offer a phase advance of $\Delta \mu_{x, y} \approx 2 \pi$. In order to minimize the maximum betatron amplitudes of protons surviving the collimation system, the longitudinal positions of collimators (the same as the phase advance between collimators) were optimized with the code DJ [29-30].

For next-generation colliders, a reasonable idea to improve the transverse collimation efficiency is to add one more collimation stage to the four-stage collimation system used at the LHC, which means a larger phase advance is needed in the transverse collimation section. On the premise of guaranteeing the beta functions without significantly increasing the total length of the collimation section, replacing warm quadrupoles by cold quadrupoles in the section is the only viable method. Next, we will explore the feasibility of this method in detail, together with the design scheme using conventional warm quadrupole magnets.

\section{B. Requirements for the lattice design for momentum collimation}

In general, a particle reaches the primary collimator with a mixing of betatron amplitude and momentum deviation. So we can define the largest momentum deviation $\delta_{\max }$ with which a particle can pass through the primary momentum collimator by the following formula [28]:

$$
\delta_{\max }=\frac{n_{1} \sqrt{\varepsilon}}{\eta_{1}},
$$

where $n_{1}$ denotes the aperture of the primary momentum collimator in units of $\sigma$ (containing the dispersive part) 
and $\varepsilon$ denotes the geometric emittance in rms, while $\eta_{1}$ denotes the normalized dispersion at the collimator. If the maximum normalized dispersion in the primary momentum collimation section is larger than the one at the DS or the whole arc section, in principle, there will be very little beam losses in the downstream DS section or even all the arc sections, based on the fact that the arc aperture is larger than $n_{1}$. For more specific considerations, the normalized dispersion at the primary momentum collimator must satisfy [31]

$$
\left|\eta_{D, \text { prim }}\left(n_{1}\right)\right| \geq \frac{n_{1} \eta_{D, \text { arc }}}{A_{\text {arc,inj }}\left(\delta_{p}=0\right)-\left(n_{2}^{2}-n_{1}^{2}\right)^{1 / 2}}
$$

to avoid cold losses at the DS or in the arc, where $A_{\text {arc,inj }}\left(\delta_{p}=0\right)$ denotes the arc aperture for on-momentum particles in units of $\sigma ; \eta_{D \text {,arc }}$ is the normalized dispersion with errors in the focusing quadrupole magnets; $n_{1}$ and $n_{2}$ denote the apertures of primary and secondary momentum collimators, respectively. In addition, to ensure that the cut of the secondary halo is independent of the particle momentum, the dispersion derivative $\eta^{\prime}{ }_{x}$ at the position of the primary momentum collimator must satisfy [28]

$$
\eta_{x}^{\prime}=0
$$

As the momentum collimation deals with much smaller halo particles than the transverse collimation does and the impact parameters at the primary momentum collimators are also much larger, the collimation efficiency is not a problem. At the LHC, the momentum collimation insertion is simpler than the transverse collimation. However, the cleaning limits for the primary off-momentum halo remain to be investigated. The single diffractive particles from the primary momentum collimators can be cleaned by the downstream collimators and do not pose problems to the DS regions.

\section{Lattice scheme I with room-temperature quadrupoles in the transverse collimation section}

In order to confirm the effectiveness of the novel method, the SPPC collimation system is used as a test bench. As shown in the Appendix, one can see that two very long straight insertions, LSS1 and LSS5, with a length of $4.3 \mathrm{~km}$ are used for collimation and extraction, respectively. In a dedicated collimation section, warm quadrupoles are usually used for their high radiation resistance. However, for very-high-energy proton beams, the focusing strength is a problem. Thus, we use quadrupole groups here with each representing several quadrupole units arranged together and acting as one quadrupole. For the momentum collimation section, in order to produce the required dispersion, four groups of cold dipoles of the arc dipole type are used. Meanwhile, cold quadrupoles are also used to control the betatron functions in the limited space. Figure 2 shows the

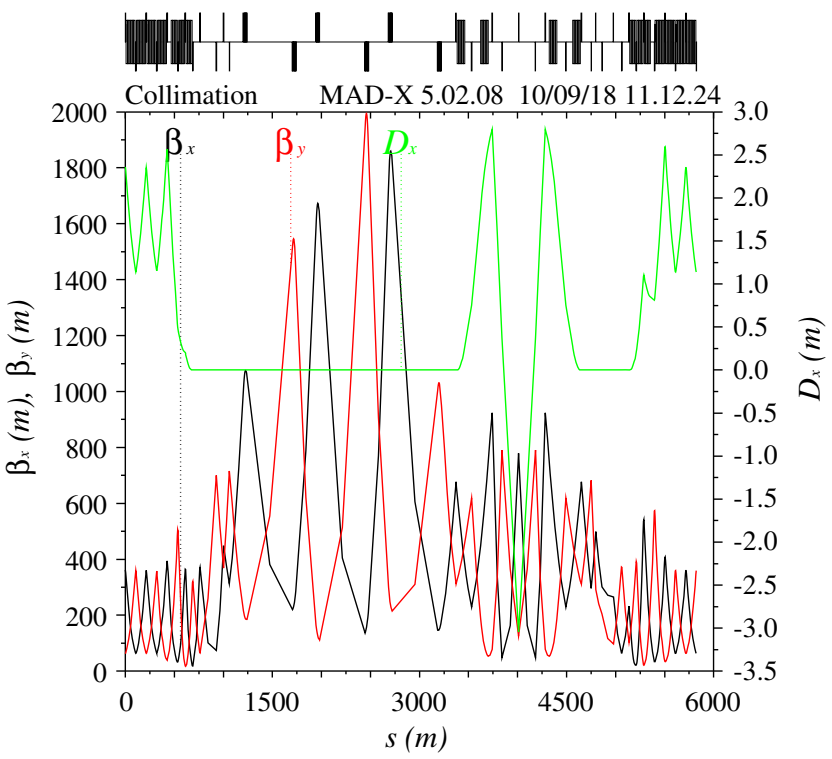

FIG. 2. The betatron and dispersive functions of the collimation insertion in lattice scheme I.

optics in lattice scheme I for the SPPC collimation system, which is similar to the lattice design in the FCC-hh to some extent $[19,32]$. The main parameters are listed in Table I.

\section{Lattice scheme II with only SC magnets}

For a multistage collimation system, the primary and secondary collimators generate secondary and tertiary halo particles that extend several $\sigma$ beyond the collimator settings, and some of them escape from the collimation insertion and are lost on the inner SC triplets at IPs where the apertures are reduced by the very large $\beta$ functions.

TABLE I. Basic parameters of the collimation insertion in lattice scheme I.

\begin{tabular}{lccc}
\hline \hline Parameter & Unit & $\begin{array}{c}\text { Betatron } \\
\text { collimation }\end{array}$ & $\begin{array}{c}\text { Momentum } \\
\text { collimation }\end{array}$ \\
\hline Insertion length & $\mathrm{km}$ & 2.55 & 1.75 \\
Maximum beta function & $\mathrm{km}$ & $1.86 / 1.99$ & $0.92 / 0.79$ \\
$\quad \beta_{x} / \beta_{y}$ & & & \\
Phase advance $\mu_{x} / \mu_{y}$ & $\mathrm{rad}$ & $1.89 \pi / 1.91 \pi$ & $2.27 \pi / 2.14 \pi$ \\
Quadrupole length & $\mathrm{m}$ & 3.3 & 6.0 \\
Maximum quadrupole & $\times 10^{-3} \mathrm{~m}^{-2}$ & 0.19 & 2.2 \\
$\quad$ & & & \\
$\quad$ strength & $\mathrm{mm}$ & 50 & $56-80$ \\
$\begin{array}{l}\text { Maximum quadrupole } \\
\quad \text { magnetic field }\end{array}$ & $\mathrm{T}$ & 0.6 & 7.8 \\
$\begin{array}{l}\text { Dipole length } \\
\text { Dipole magnetic field }\end{array}$ & $\mathrm{m}$ & $\ldots$ & 14.45 \\
$\begin{array}{l}\text { Dipole aperture } \\
\text { Maximum normalized }\end{array}$ & $\mathrm{T}$ & $\ldots$ & 12 \\
$\quad \mathrm{~mm}$ & $\ldots$ & 50 \\
$\quad$ dispersion & & $\ldots$ & -0.124 \\
\hline \hline
\end{tabular}




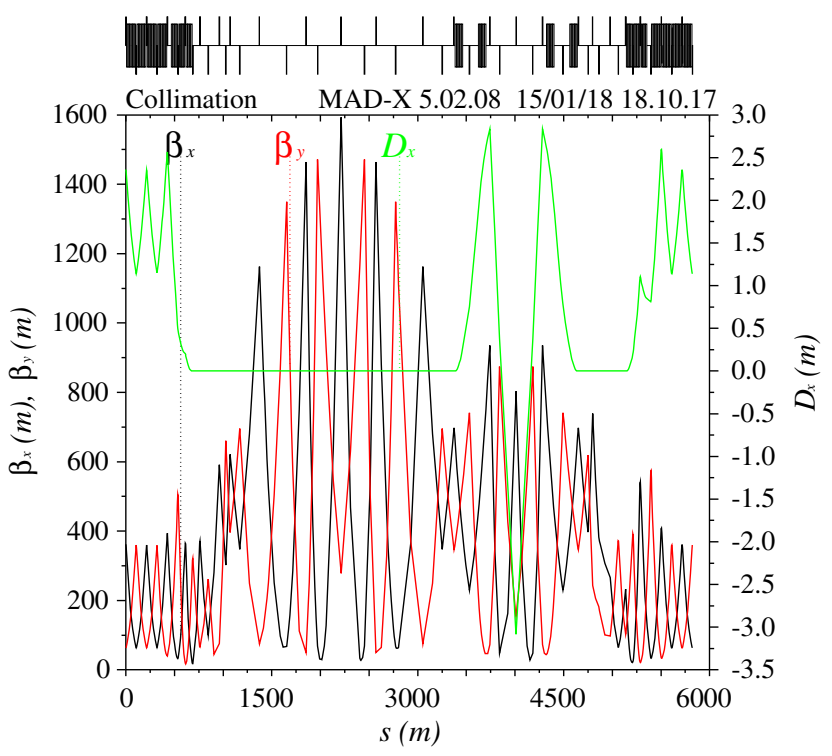

FIG. 3. The betatron and dispersive functions in the collimation insertion in lattice scheme II.

At the LHC, in order to locally provide additional protection from the tertiary halo [33], 16 tertiary collimators in pairs are installed at each side of the four experiment insertions. These tertiary collimators also can protect the triplets from miskicked beams, for example, due to asynchronous kicking for beam dumping and failures of the normal conducting separation magnets [34]. One source of the machine-induced backgrounds at the detectors is due to the upstream interaction of beam protons with residual gas molecules or collimators. According to the study at the LHC [35-36], the beam-gas interaction is the main contribution of the background, higher than the beam halo by one order of magnitude.

For the SPPC, the stored energy in the beam is as high as 9.1 GJ per beam, about 25 times that of the LHC at design energy, and the development of hadronic and
TABLE II. Basic parameters of the collimation insertion in lattice scheme II.

\begin{tabular}{lccc}
\hline \hline Parameter & Unit & $\begin{array}{c}\text { Betatron } \\
\text { collimation }\end{array}$ & $\begin{array}{c}\text { Momentum } \\
\text { collimation }\end{array}$ \\
\hline Section length & $\mathrm{km}$ & 2.55 & 1.75 \\
$\begin{array}{l}\text { Maximum beta function } \\
\quad \beta_{x} / \beta_{y}\end{array}$ & $\mathrm{~km}$ & $1.59 / 1.47$ & $0.93 / 0.87$ \\
Phase advance $\mu_{x} / \mu_{y}$ & $\mathrm{rad}$ & $3.54 \pi / 3.31 \pi$ & $2.27 \pi / 2.14 \pi$ \\
Quadrupole length & $\mathrm{m}$ & 6.0 & 6.0 \\
$\begin{array}{l}\text { Maximum quadrupole } \\
\text { strength }\end{array}$ & $\times 10^{-3} \mathrm{~m}^{-2}$ & 1.8 & 2.2 \\
Quadrupole aperture & $\mathrm{mm}$ & $70-80$ & $56-80$ \\
Maximum quadrupole & $\mathrm{T}$ & 8.0 & 7.8 \\
$\quad$ magnetic field & & & \\
$\begin{array}{l}\text { Dipole length } \\
\text { Dipole magnetic field }\end{array}$ & $\mathrm{m}$ & $\ldots$ & 14.45 \\
$\begin{array}{l}\text { Dipole aperture } \\
\text { Maximum normalized }\end{array}$ & $\mathrm{Tm}$ & $\ldots$ & 12 \\
$\quad$ dispersion & $\mathrm{m}^{1 / 2}$ & $\ldots$ & -0.124 \\
\hline \hline
\end{tabular}

electromagnetic showers becomes more intense due to higher proton energy. It is foreseeable that the tertiary halo in the machine will be much more severe. One more stage of collimators installed in the transverse collimation section will convert the tertiary beam halo into a quaternary beam halo, thus helping to dilute the halo particles in the experiments and reduce the risk of quenching in SC inner triplets, and the experimental background level may be reduced more or less. However, when warm quadrupoles are used, there is not enough phase advance to add additional collimators due to the weak focusing strength, or significant space will have to be added. As space is so precious, we try to apply SC quadrupoles in the transverse collimation section to create more focusing cells. These quadrupoles are very different from those in the arcs; they will be designed with enlarged apertures and a lower pole strength (not higher than $8 \mathrm{~T}$ ) and are somewhat

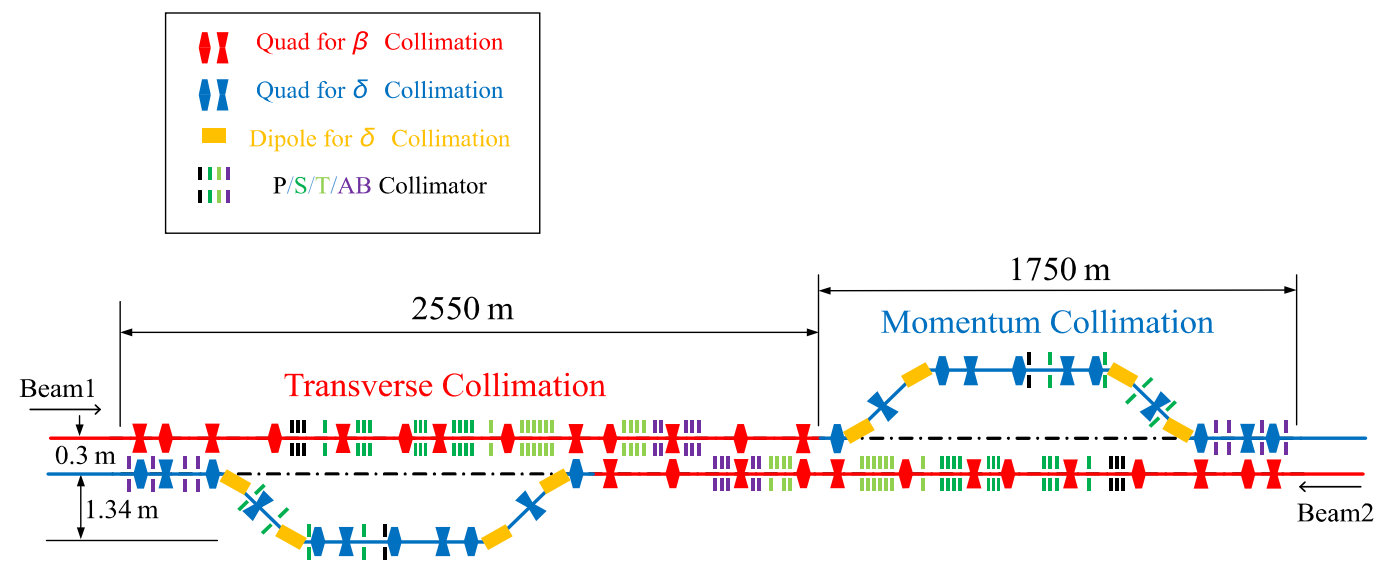

FIG. 4. Layout of the collimation insertion. $P, S, T$, and AB denote the primary collimator, secondary collimator, tertiary collimator, and absorber, respectively. 
comparable to the triplet quadrupoles used in the experiment insertions at the LHC. In this way, much higher transverse collimation efficiency can be obtained, so the probability of particle losses in the downstream momentum collimation section and the residual halo at the experiments will be reduced largely. The phase advance between the secondary and tertiary collimators should be similar to the one between the primary and secondary collimators, assuming that most of the tertiary beam halo particles are emitted from the secondary collimators. Figure 3 shows the lattice functions. The main parameters are listed in Table II.

The same as for lattice scheme I, we also need to consider the two collimation systems for each beam in one insertion. The distance between two beams is set to about $30 \mathrm{~cm}$ at the arcs, which is considered to be enough to install one collimator for one beam but cannot accommodate an additional collimator at the same location for another beam. In the momentum collimation section, the horizontal separation from the other beam is enlarged to $1.64 \mathrm{~m}$, which will allow the installation of the collimators for the two beams. Meanwhile, we apply SC quadrupoles with twin apertures for the two beams in the overlapping region with nominal separation. The layout of the collimation section is shown in Fig. 4.

\section{SIMULATION RESULTS OF THE SPPC COLLIMATION SYSTEM}

\section{A. Collimation inefficiency}

To quantify the performance of the collimation system more precisely, the local cleaning inefficiency $\tilde{\eta}_{c}$, which is the ratio of the number $N_{i}$ of lost protons at any location of the ring in a given bin of length $L_{i}$ (set to $10 \mathrm{~cm}$, in general) over the total number $N_{\text {tot }}$ of lost protons [4], is calculated:

$$
\tilde{\eta}_{c}=\frac{N_{i}}{N_{\text {tot }} \cdot L_{i}}
$$

For slow and continuous losses, the circulating intensity in the machine can be described as a function of time $t$ :

$$
N(t)=N_{0} \exp \left(-\frac{t}{\tau}\right)
$$

where $N_{0}$ is the nominal intensity and $\tau$ is the finite beam lifetime. At the LHC, in order to ensure commissioning and performance in nominal running, conservative minimum lifetimes $\tau_{\min }$ are assumed as $0.2 \mathrm{hr}$ at the top energy and $0.1 \mathrm{hr}$ at the injection energy [37]. For an operation with the minimum beam lifetime $\tau_{\min }$, the total intensity $N_{\text {tot }}^{q}$ is limited by the quench limit $R_{q}$ :

$$
N_{\mathrm{tot}}^{q}=\frac{\tau_{\min } \cdot R_{q}}{\tilde{\eta}_{c}},
$$

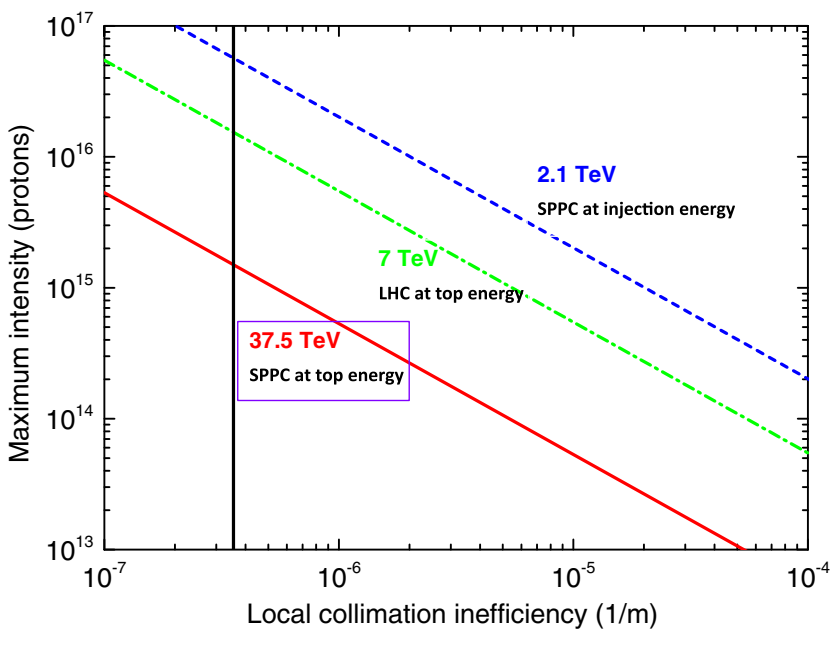

FIG. 5. The maximum total intensity is shown as a function of the local cleaning inefficiency for SPPC injection, top energy, and the LHC top energy.

where $\tilde{\eta}_{c}$ is the local cleaning inefficiency as defined in Eq. (5) and the quench limit $R_{q}$ in units of protons $/ \mathrm{m} / \mathrm{s}$ is related to the transmission capability and the maximum deposited energy density, which defines the allowed maximum local proton loss rates [38]. Figure 5 shows the maximum total intensity at the quench limit as a function of the local cleaning inefficiency, assuming that minimum beam lifetimes of $0.1 \mathrm{hr}$ at the injection energy and $0.2 \mathrm{hr}$ at the top energy must be satisfied just like at the LHC. In the baseline design of the SPPC, the SC magnets in the arcs use the full iron-based high temperature superconducting (HTS) technology [39], and the field strength of the main dipoles is $12 \mathrm{~T}$. However, as the magnet technology is still being developed [40], the quench limit is not yet available. In this article, the quench limit value $R_{q}$ for the SPPC arc magnets is estimated by the following formula [41]:

$$
R_{q}=1.7 \times 10^{8} E^{-(3 / 2)},
$$

where the energy $E$ is in units of $\mathrm{TeV}$. The same scaling was applied in the FCC-hh design [19] from the NbTi technology at the LHC to the $\mathrm{Nb}_{3} \mathrm{Sn}$ technology at the FCC-hh, assuming the same quench level of $5 \mathrm{~mW} / \mathrm{cm}^{3}$ $[38,42]$. Thus, the quench limit $R_{q}$ is estimated as $0.74 \times 10^{6}$ protons $/ \mathrm{m} / \mathrm{s}$ at the top energy $37.5 \mathrm{TeV}$ and $0.56 \times 10^{8}$ protons $/ \mathrm{m} / \mathrm{s}$ at the injection energy $2.1 \mathrm{TeV}$. From Fig. 5, it is noted that the SPPC at the top energy has the most stringent requirements of cleaning inefficiency, where the vertical line in black is for the design goal of this collimation study, which means that the nominal intensity of $1.5 \times 10^{15}$ protons per beam at the top energy requires a cleaning inefficiency of $3.55 \times 10^{-7} \mathrm{~m}^{-1}$, that is more stringent than that at the LHC by about one order. 
TABLE III. Collimator parameters for the SPPC.

\begin{tabular}{lccccc}
\hline \hline $\begin{array}{l}\text { Collimator } \\
\text { acronym }\end{array}$ & $\begin{array}{c}\text { Length } \\
(\mathrm{m})\end{array}$ & Number & $(\sigma)$ & Material & $\begin{array}{c}\text { Lattice } \\
\text { schemes }\end{array}$ \\
\hline TPC & 0.6 & 3 & 6 & Carbon & I, II \\
TSC & 1.0 & 11 & 7 & Carbon & I, II \\
TTC & 1.0 & 11 & 8.3 & Copper & II \\
TAB & 1.0 & 5 & 10 & Tungsten & I, II \\
TQC & 1.0 & 4 & 10 & Tungsten & I, II \\
MPC & 0.6 & 1 & 12 & Carbon & I, II \\
MSC & 1.0 & 4 & 15.6 & Carbon & I, II \\
MAB & 1.0 & 4 & 17.6 & Tungsten & I, II \\
\hline \hline
\end{tabular}

\section{B. Simulation results of the beam loss distributions}

Multiparticle simulations using the two lattice schemes described in Secs. II C and II D have been carried out with the code MERLIN, which is a $\mathrm{C}++_{+}$accelerator library easily to extend and modify. Its organization structure can be found in Ref. [43]. This code has good agreement with the well-known collimation version SixTrack $+\mathrm{K} 2$ after the benchmarked work [44]. In the code, protons are considered lost if they undergo an inelastic interaction within the collimator jaws or if they intercept the mechanical beam pipe. The local cleaning inefficiency $\tilde{\eta}_{c}$ is used as the measure of the performance for collimation simulations.

Besides the arc sections, only the functional lattices for the collimation and experiment insertions have been used and all the other insertions, such as rf, injection, and extraction insertions, are replaced by periodic FODO structures. The physical aperture in the arcs is set to be the inner aperture of the beam screen [45] that is used to absorb the synchrotron radiation, and its cross section is a superposition of an ellipse and a rectangle with a mean radius of about $15 \mathrm{~mm}$. As for the transverse collimation section, the apertures of the warm quadrupoles are $60 \mathrm{~mm}$ or larger than $85 \sigma$ in lattice scheme I, and the cold quadrupole apertures are enlarged to $70-80 \mathrm{~mm}$ or larger than $130 \sigma$ in lattice scheme II, which are about the same as the triplet magnet apertures in the experimental regions. In the momentum collimation section, the quadrupole magnet aperture is enlarged slightly on the premise that the pole magnetic field does not exceed the preset value of $8 \mathrm{~T}$.

The collimator parameters in the simulations are shown in Table III, where $T$ is for transverse, $M$ for momentum, $P$ for primary, $S$ for secondary, the second $T$ for tertiary, $Q$ for quaternary, $\mathrm{AB}$ for absorber, and $C$ for collimator. The collimator settings are quoted from the LHC design settings [35] and run I operational settings [46]. The locations of the four-stage collimators in the collimation insertion are shown in Fig. 4. As the first approach for the transverse collimator settings, the physical gaps and phase advances are set as the same as the ones at the LHC for just verifying the effectiveness of the collimation method, especially in cleaning particles related to the single diffractive effect. The primary momentum collimators are placed just downstream of the middle quadrupole between the second and third groups of dipoles to satisfy Eqs. (3) and (4), where the normalized dispersion is close to the maximum. The other momentum collimators are placed further downstream with
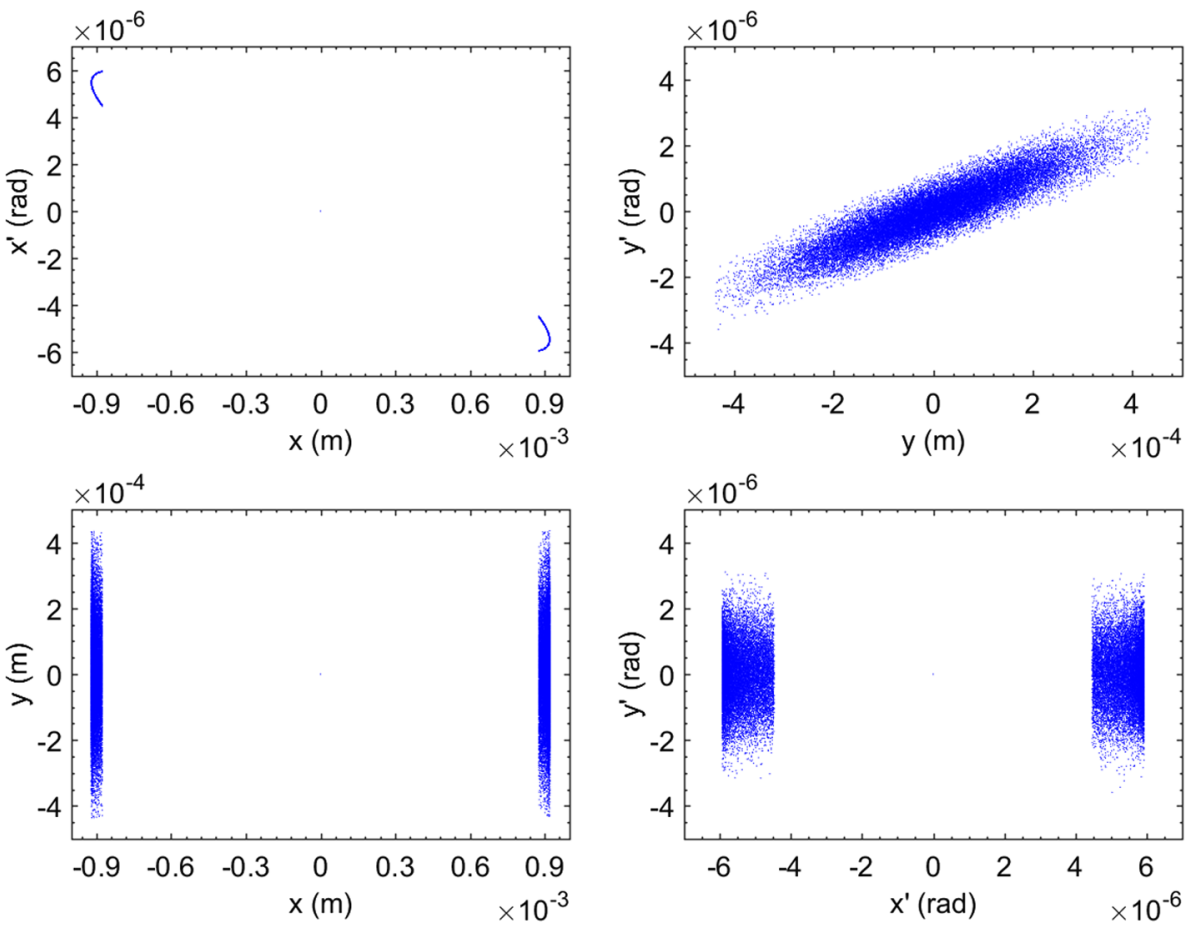

FIG. 6. Initial horizontal halo distribution for collimation simulations. 

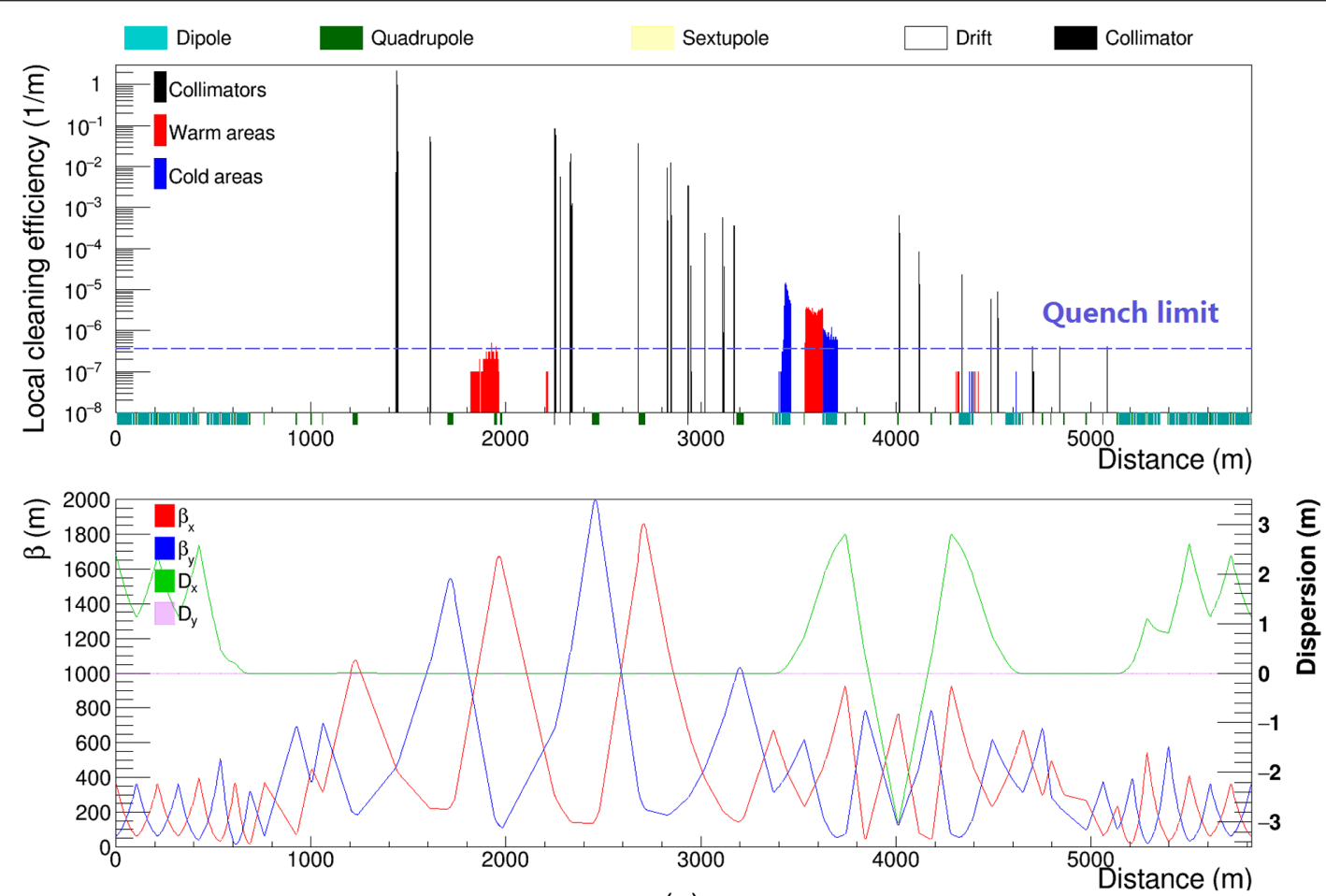

(a)
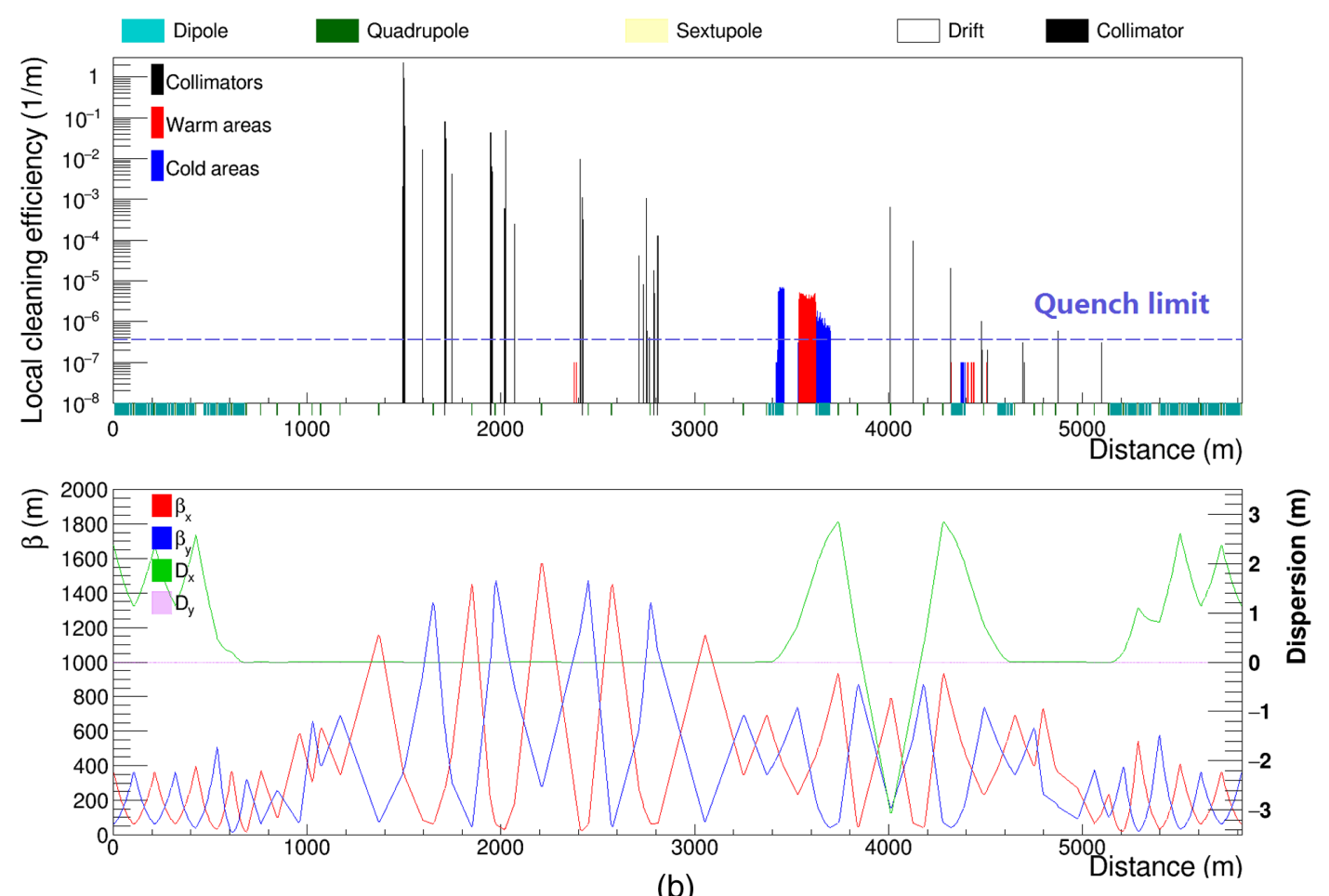

(b)

FIG. 7. Proton loss maps in the collimation insertion with an initial horizontal halo distribution, using lattice scheme I (a) and II (b).

the same phase advances as used in the current LHC momentum collimation. Eleven tertiary collimators have been added in lattice scheme II, but the jaw locations and orientations have not been optimized. In addition, two quaternary collimators are installed before the inner triplet magnets at each experimental region, which are to intercept the residual halo and to protect the IR bottlenecks.

In order to increase the accuracy of calculating the local cleaning inefficiency, 100 million protons are tracked for 300 turns in the SPPC ring, in which the initial beam 

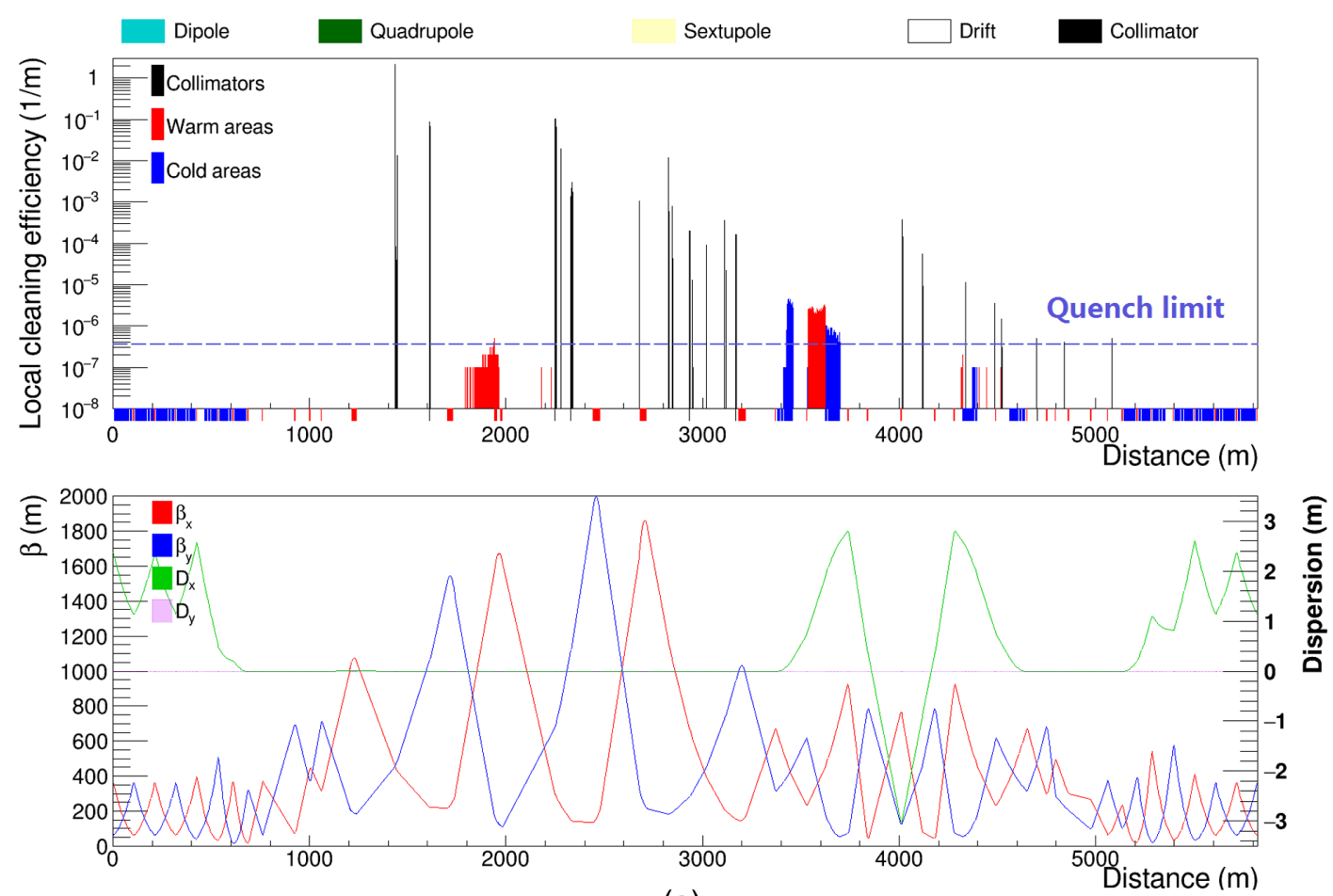

(a)
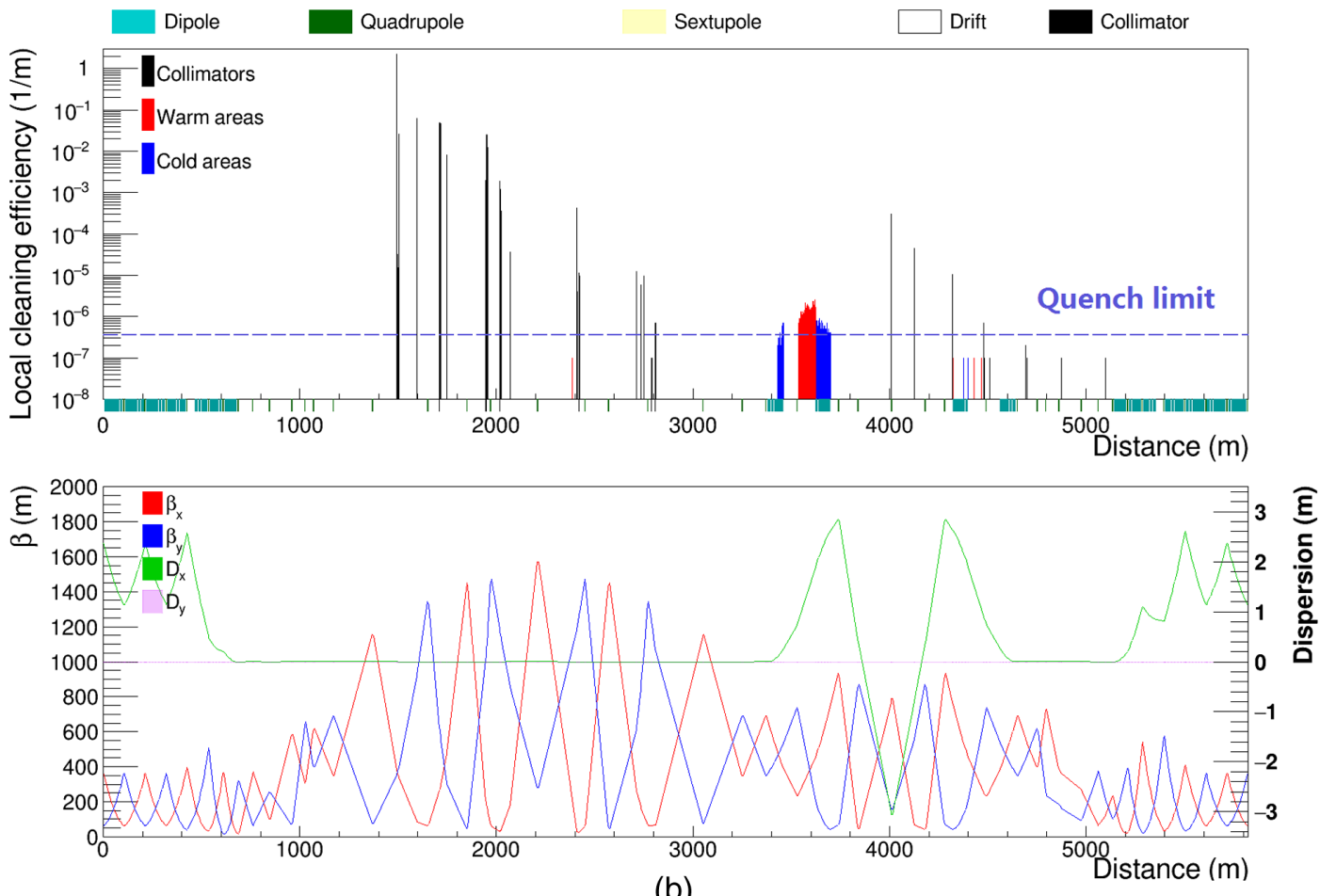

(b)

FIG. 8. Proton loss maps in the collimation insertion with an initial vertical halo distribution, using lattice scheme I (a) and II (b).

distribution is represented by the so-called halo distributions for saving computing time. For example, for the horizontal halo collimation, the horizontal distribution is presented by two short arcs with a radius being the TPC half-gap, and the vertical distribution is a cut Gaussian at
$3 \sigma$, just as shown in Fig. 6. The impact parameter at the primary collimators is chosen as $1 \mu \mathrm{m}$ for negligible emittance growth from the previous turn, pending such a study so the LHC assumption is used [41]. Based on the above parameter settings, the simulations are carried out 


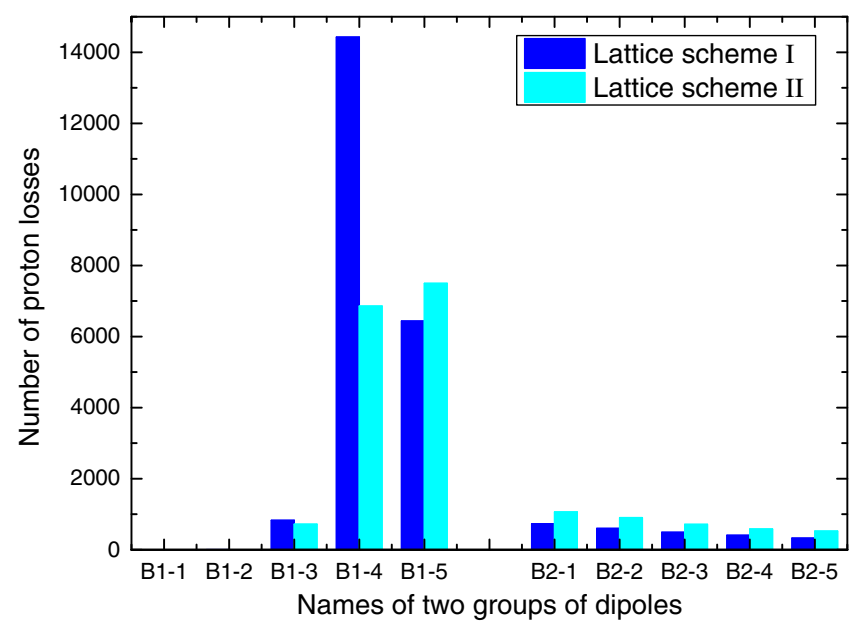

(a)



(b)

FIG. 9. Proton losses in the first and second groups of dipoles with two lattice schemes, with an initial horizontal (a) or vertical (b) halo distribution. The total particles in the simulations are 100 million.

for both the horizontal and vertical halo collimations. This simulation method and assumptions can maintain a good computing performance, which has been illustrated in Refs. [41,46-47].

Figures 7 and 8 show the proton loss distribution in the collimation insertion using lattice schemes I and II, with the initial horizontal and vertical halo distributions, respectively. One can see that there are still cold-area losses at the dipoles where the dispersion starts to rise, which can be foreseen due to the protons with large momentum deviations, related to the single diffractive effect. One can find the detailed comparison of proton losses in the first and second groups of cold dipoles with different lattice design schemes and initial halo distribution in Fig. 9.

With an initial horizontal halo distribution, the proton losses can be reduced to half by introducing 11 tertiary collimators, but it could still lead to cold dipole quenches if no further protection measures are taken. In contrast, with initial vertical halo distribution, the tertiary collimators can reduce the proton losses by about one order of magnitude.

As shown in Fig. 7, for an initial horizontal halo distribution, one will see important proton losses at the cold dipoles due to the single diffractive effect, even with the help of tertiary collimators. To solve the problem, some protective collimators (used as absorbers) can be placed here. Different from the arc DS regions where the lattice structure is very strict and the space is very tight, it is much easier to provide the space for the collimators at room temperature in the momentum collimation section. These collimators intercept the particles with large momentum deviations by large impact parameters, thus having a good cleaning efficiency. The particles with medium momentum deviations that represent a small fraction of the single diffractive particles and those scattered from the collimators will be cleaned by the downstream multistage momentum collimation system. According to the positions of the lost particles, three protective collimators in tungsten with an aperture of $10 \sigma$ and a length of $1 \mathrm{~m}$, the same as the one of the absorbers in the transverse collimation section, are placed there to intercept the particles related to the single diffractive effect. The specific locations are as follows: One protective collimator is placed between the third and fourth dipole magnets of the first dipole group to intercept particles with a very large momentum deviation, and the cryostat for the dipole group is split into two parts to allow the insertion of the collimator at room temperature; another one is placed before the quadrupole between the first and second groups of dipoles to protect the quadrupole, and the third one is placed in front of the second group of dipole magnets. Figure 10 shows the beam loss distribution in the collimation insertion with three protective collimators, with the initial horizontal halo distribution; nearly all the proton losses in the cold regions disappear. Almost all the lost protons are in the collimators, and the majority of single-diffractive protons escaping the betatron cleaning are intercepted by the protective collimators at dispersive locations upstream of the multistage momentum cleaning hierarchy.

As mentioned earlier, the beam losses in the experimental regions are also a major concern. According to the simulation results, the tertiary collimators can intercept the tertiary halo effectively; as evidence, the proton losses at the quaternary collimators are reduced by more than one order in the experimental region LSS7 and by 4 times in the experimental region LSS3, compared to the lattice scheme I, and the results are shown in Fig. 11. This means it is much helpful to reduce the residual halo particles in the experimental regions by adding one more stage collimators in the transverse collimation section. 

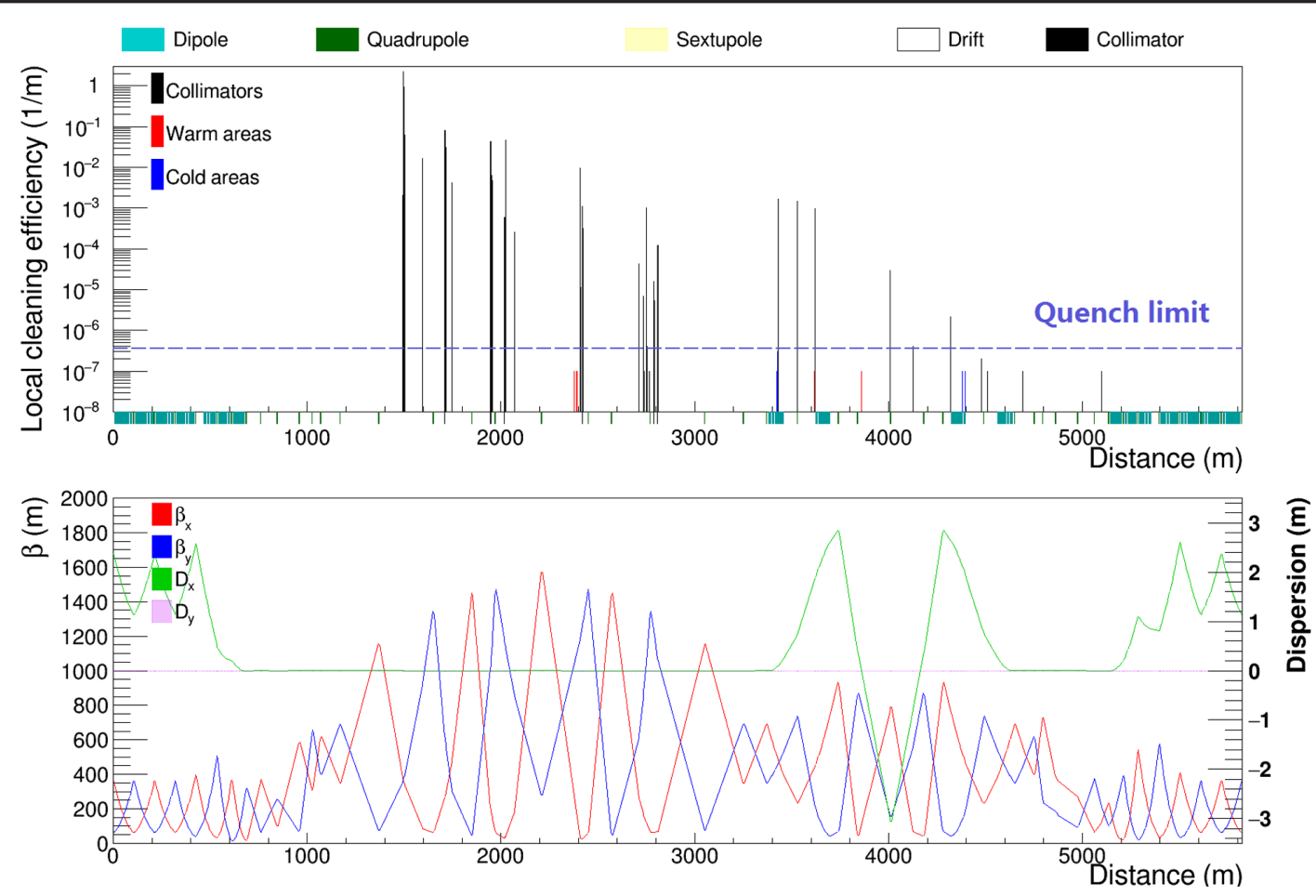

FIG. 10. The proton loss distribution of the cleaning insertion with protective collimators.

\section{ANALYSIS OF THE ENERGY DEPOSITION IN THE SC MAGNETS OF THE COLLIMATION SECTION}

\section{A. Quench limits}

When a high-energy proton interacts with dense matter, the development of hadronic and electromagnetic cascades is the main process of energy deposition, which produces thousands of low-energy particles continuously until all of them are stopped in the matter and absorbed. These processes occur in the interactions between the primary protons and collimators or vacuum chamber. If these secondary showers deposit energy in the SC magnet coils, then the local energy or power deposition may exceed the quench limit value, and the $\mathrm{SC}$ magnets will experience a quench, from the SC state to the normal conducting state [48]. In general, the quench limit is a function of the local magnetic field, geometrical loss pattern, operating temperature, cooling conductions, and time distribution of beam losses [49]. In order to protect the SC magnets in the collimation section from quenches, it is very important to shield the particle showers and reduce the energy or power deposition in the magnet coils. In this section, we provide the protection schemes for the SC quadrupoles and dipoles which are used in the collimation section of lattice scheme II. For simplicity, only steady state beam loss is considered, and the heat in the coils is constantly removed by the helium bath through the cable insulation [49].

To reduce the energy deposition in the SC coils, the cold quadrupoles in the transverse collimation section are designed with an enlarged aperture and a lower magnet field. On the one hand, the larger aperture means larger acceptance for the magnet to intercept as few as possible particles; on the other hand, the quench limit increases as the magnetic field decreases. As shown in Table II, the highest pole-tip magnetic field is $8 \mathrm{~T}$, which is lower than the IR quadrupoles at the LHC. Considering the helium II and helium boiling heat transfer mechanisms, which allow extracting more heat from the cable than the only solid conducting through the cable insulation, the estimated quench limit in the cable of cold quadrupoles ranges from 50 to $100 \mathrm{~mW} / \mathrm{cm}^{3}$ [49-50].

For the SC dipoles used in the momentum collimation section, the magnetic field is $12 \mathrm{~T}$, which will use full ironbased HTS technology in the SPPC. However, some physical properties of the cable have yet to be determined so far. Thus, the conservative estimate of quench limits in the cable of cold dipoles is $5-10 \mathrm{~mW} / \mathrm{cm}^{3}$ [51], just the same as the $\mathrm{Nb}_{3} \mathrm{Sn}$ cable.

\section{B. Energy deposition in the SC quadrupoles}

The Monte Carlo analysis process for energy or power deposition includes the following steps. (i) The initial beam halo distribution is generated with the MERLIN code, which records the coordinate information of the halo protons. These recorded data provide an input distribution for shower and energy deposition studies with the FLUKA code [52-53]. (ii) Particle-shower simulations with FLUKA are carried out to estimate the energy deposition in cold magnets for lost primary protons. 

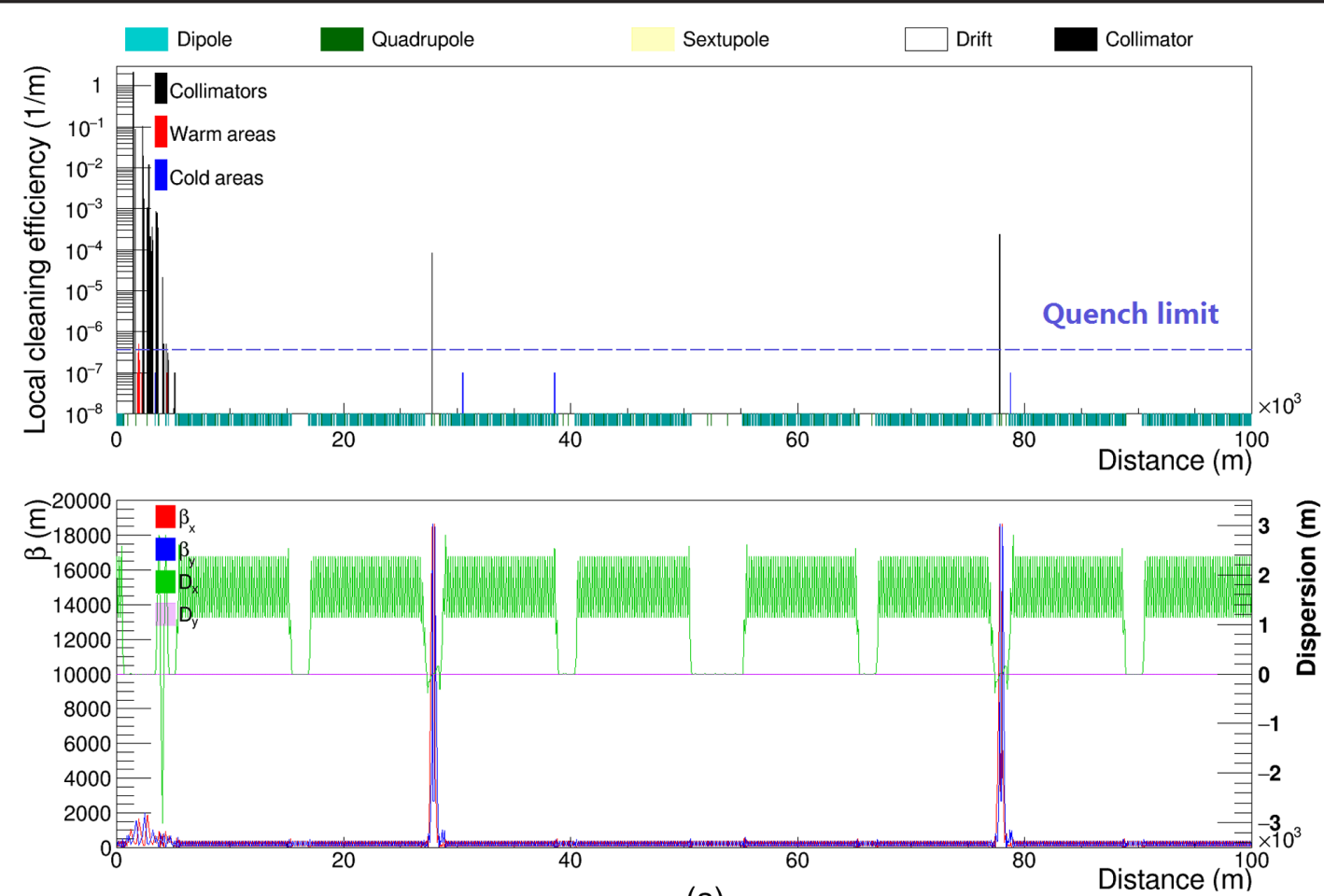

(a)


FIG. 11. Proton loss distribution along the full ring in lattice scheme I (a) and II (b), with initial vertical halo distribution.

(iii) The power depositions in the most critical position of the coils for different beam loss scenarios are calculated. According to the tracking results with MERLIN, we know that $88 \%$ of the protons are lost in the first turn; for the calculation results with FLUKA, the power deposition per bin is divided by $88 \%$ to take into account the lost particles in the subsequent turns. The quench probability is evaluated based on the power deposition.

For power deposition evaluation in the SC quadrupoles in the transverse collimation section, observing the proton loss distribution as shown in Fig. 10, the first quadrupole downstream of the primary collimators is considered as 


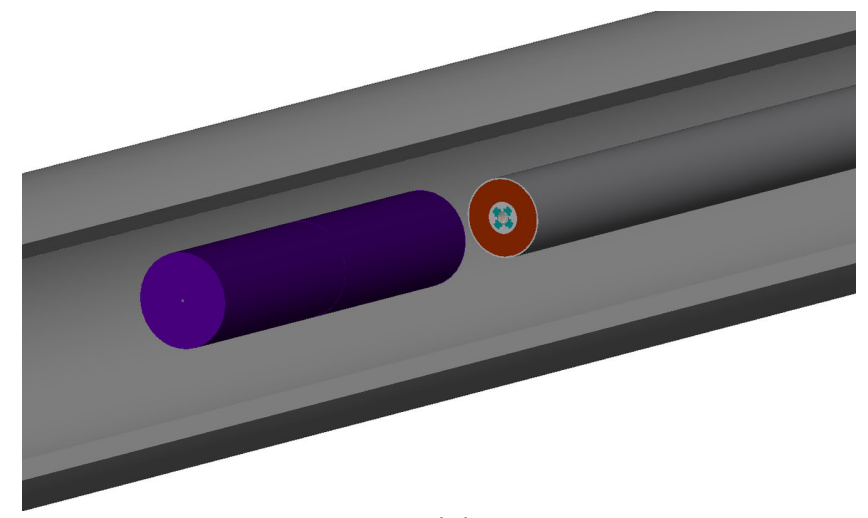

(a)

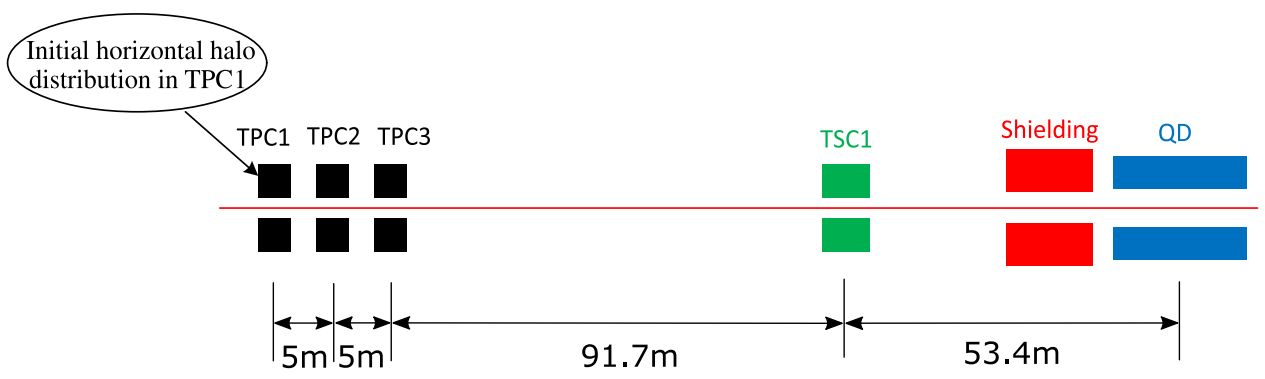

(b)

FIG. 12. Geometrical model of the shielding and defocusing quadrupole (a) and the positions of elements in FLUKA (b) for an analysis of energy deposition in the first SC quadrupole after the primary transverse collimators, where the magnetic fields are not included in the simulation.

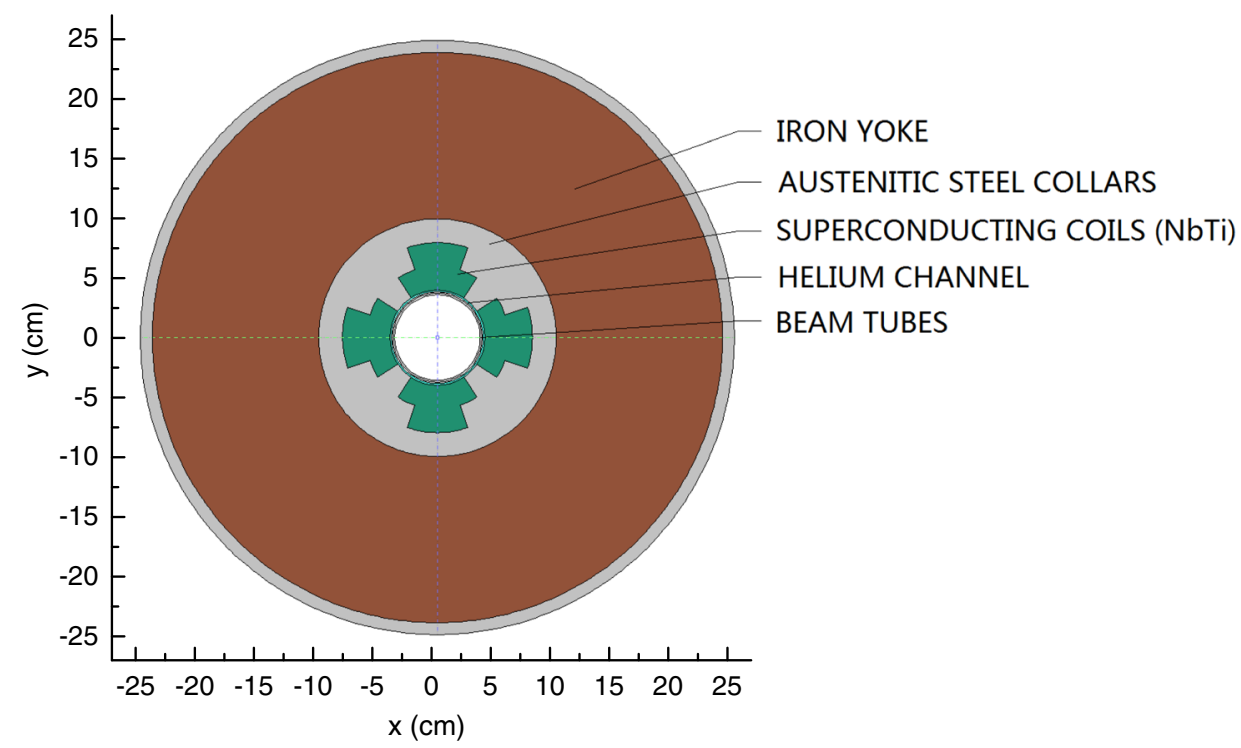

FIG. 13. Cross section of the SC quadrupole in the transverse collimation section.

the magnet which bears the greatest risk of quench. Thus, the shower produced by the interaction between the halo and upstream collimators, including three primary collimators and one secondary collimator, is regarded as the main source of energy deposition in the cold coils of the quadrupole. The layout of a geometry model is shown in Fig. 12, where the input distribution is provided by the MERLIN code with the initial horizontal halo distribution.

As mentioned in Sec. III B, in order to reduce the probability of particle losses in the SC coil of quadrupoles, 


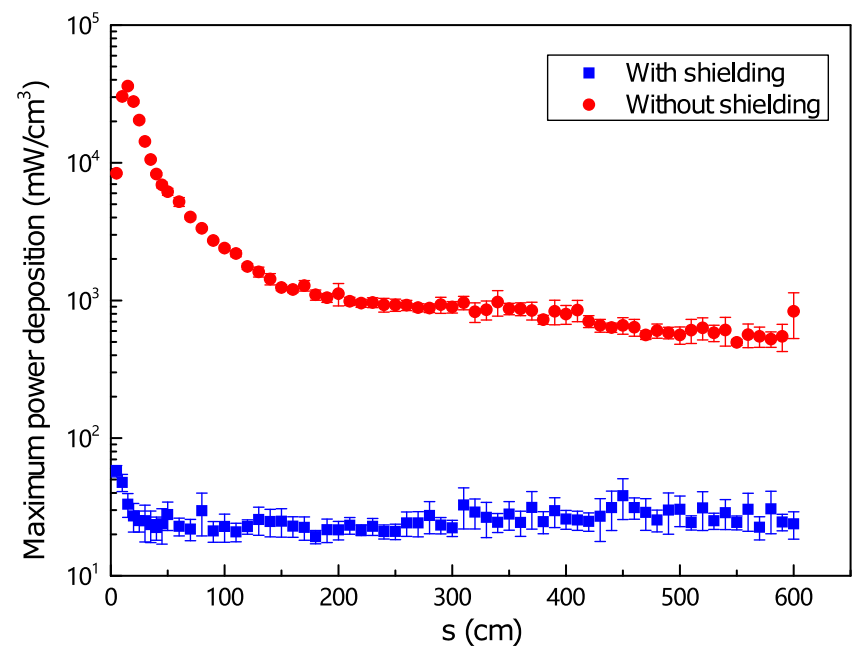

FIG. 14. Maximum power deposition density along the QD after the primary collimators.

they are designed with a wider aperture, and for the case of QD in Fig. 12 it is $80 \mathrm{~mm}$. The material of coils is a mixture of 50\% niobium-titanium (NbTi) and 50\% copper. The cross section of the SC quadrupoles used in the transverse collimation section is shown in Fig. 13, referring to the insertion region wide aperture quadrupoles at the LHC [48].

As a high- $Z$ material, tungsten has been chosen as the shielding material, which can absorb the particle shower more effectively. In the geometry model, the shielding is placed at $1 \mathrm{~m}$ in front of the quadrupole, which is a hollow cylinder, with a length of $3 \mathrm{~m}$ and the inner half-aperture of $10 \mathrm{~mm}$ or about $37 \sigma$, and the outer radius is set to be $300 \mathrm{~mm}$ to cover the yoke of the SC quadrupole. This has proven to be tight enough to intercept the particle shower but wide enough not to violate the multistage collimation hierarchy.

Referring to the energy deposition study for the FCC-hh [26], with the assumption that the total beam is lost on the collimation system within a time period of $0.2 \mathrm{hr}$ that is used for designing the LHC collimators, the maximum power on the dogleg warm dipoles is up to $1.1 \mathrm{MW}$, which is considered to be too high to cool the dipoles easily. There is a similar situation at the SPPC. In this study, the loss rate of total beam power in one hour in the collimators is used to calculate the power deposition. This abnormal beam loss usually triggers the beam ejection mechanism into the external beam dump in a very short period that is to be determined in future studies, e.g., less than $1 \mathrm{~s}$. Faster beam loss means a shorter time to abort the beam to the beam dump. Figure 14 shows the results of the maximum power deposition density, where the bins are chosen as a compromise between calculation precision and time consumption: $0.5 \mathrm{~cm}$ in radius, $2^{\circ}$ in azimuth, and $5-10 \mathrm{~cm}$ in length (small bins for the region where the gradient is large) along the defocusing quadrupole (QD). According to the calculation results by FLUKA, the total power on the shielding is

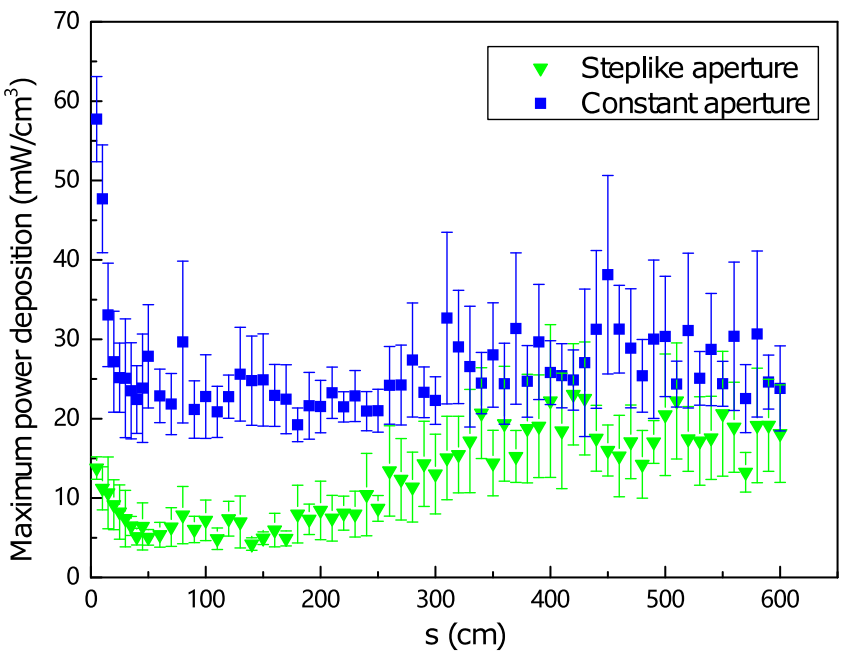

FIG. 15. Maximum power deposition along the QD before and after the aperture optimization of the shielding.

up to $480 \mathrm{~kW}$, and the peak power density is about $1.3 \mathrm{~kW} / \mathrm{cm}^{3}$, which is located in the front face of the shielding and similar to that in the passive absorbers for the FCC-hh design [25-26]. Though tungsten has a good thermal conductivity, this value may be too high to bear even for a short period, e.g., a few seconds; further optimization studies on the shielding material and structure, including the cooling system for the shielding, should be done in the future.

Compared to the case without shielding (red line), the maximum power deposition along the QD with shielding is reduced by 3 orders of magnitude (blue line). In the shielding cases, the peak power deposition is located at the first bin or the entrance part of the QD, with a value of $57.7 \mathrm{~mW} / \mathrm{cm}^{3}$. A possible reason for the peak is that it comes from the shower emerging from the end part of the shielding block. An optimized method is to slightly increase the aperture of the rear half shielding, so-called steplike shielding. Figure 15 shows the simulation results after the optimization, where the aperture of the rear half shielding is enlarged from 10 to $10.5 \mathrm{~mm}$ for the steplike shielding. One can see that with the steplike aperture the power deposition is reduced to below $30 \mathrm{~mW} / \mathrm{cm}^{3}$, which is safe from the quench limit value of $50-100 \mathrm{~mW} / \mathrm{cm}^{3}$.

\section{Energy deposition in the SC dipoles}

For the evaluation of the quenching risk in the SC dipoles used in the collimation system, the fourth dipole of the first group of dipoles, which is the closest to the first protective collimator, is considered the cold dipole which bears the highest dose of radiation. Figure 16 shows the 3D geometry model in FLUKA and the cross section of the SC dipoles, where the material of coils is chosen as a mixture of 50\% silver and $50 \% \mathrm{SmAsFeO}_{0.2} \mathrm{~F}_{0.8}$, which is one type of ironbased wire [54]. The input distribution of protons used in 

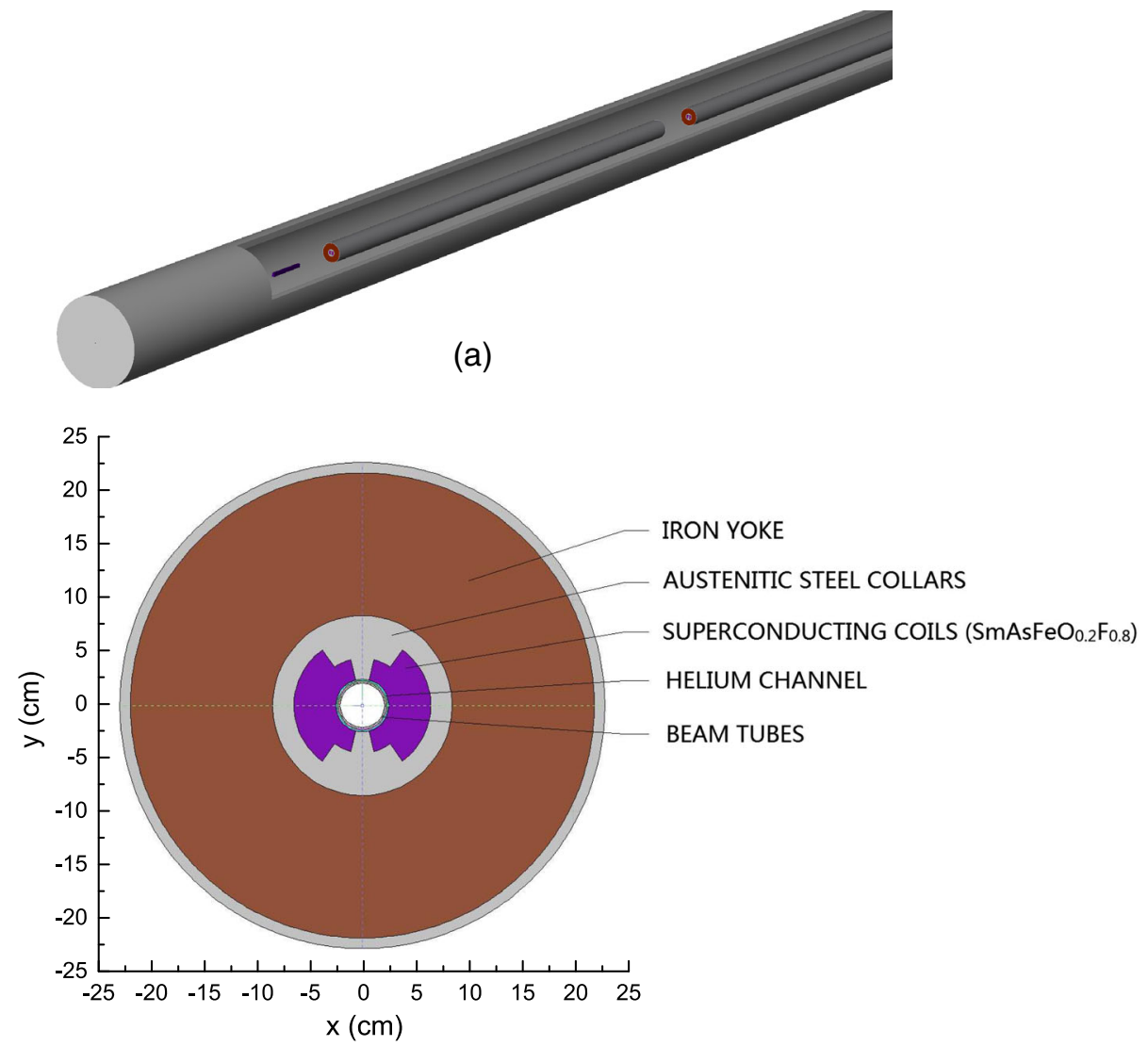

(b)

FIG. 16. A 3D geometry model in FLUKA including the first protective collimator and two following SC dipoles B1-4 and B1-5 (a) and the cross section of the SC dipoles in the momentum collimation section (b), where the magnetic fields are not included.

FLUKA is provided by the code MERLIN, which records the coordinate information of the protons lost in the first protective collimator. Here the upstream shower is not

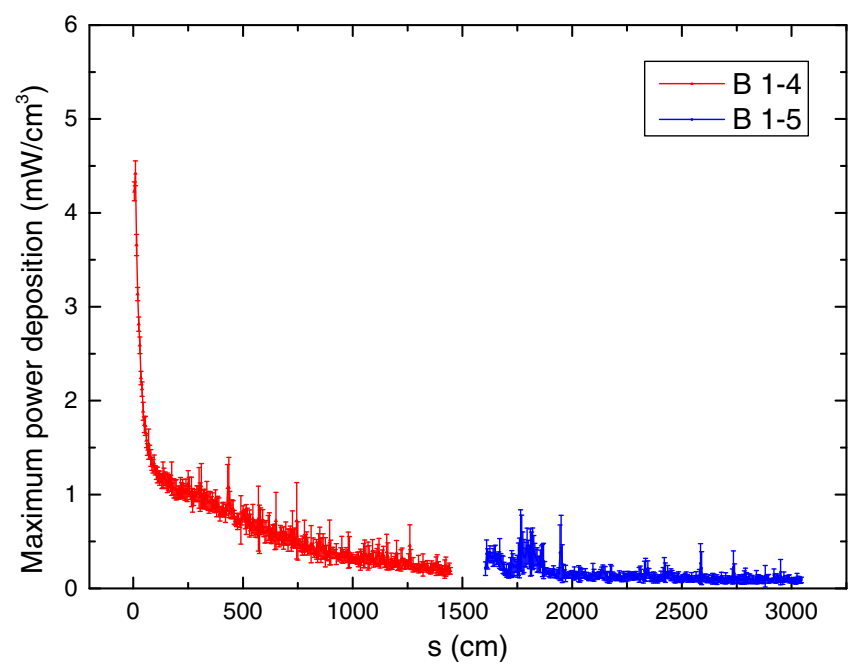

FIG. 17. Maximum power deposition along the two most exposed cold dipoles, which are closest to the first protective collimator. included, which is considered to be cleaned off by the transverse collimators and absorbers. For a one hour beam lifetime, the power load on this protective collimator is $0.9 \mathrm{~kW}$. Figure 17 shows the result of power deposition in the coils along the two most exposed dipoles, B1-4 and $\mathrm{B} 1-5$.

The maximum power deposition is $4.5 \mathrm{~mW} / \mathrm{cm}^{3}$, which is below the quench limit of $5-10 \mathrm{~mW} / \mathrm{cm}^{3}$. At present, additional shielding following the protective collimator for the SC coils is not necessary. However, this issue needs to be reconsidered for a shorter beam lifetime or possible upgrading plane; some related results have been carried out in the FCC-hh [55].

\section{CONCLUSIONS}

A novel collimation method for future proton-proton colliders is proposed, which arranges both transverse and momentum collimation systems in the same cleaning insertion and employs SC quadrupoles in the transverse collimation section. The protective collimators at the SC dipoles and the multistage momentum collimation system are integrated together, with the former to clean the single diffractive particles with large momentum deviations and 
the latter to clean the particles with medium momentum deviations and those scattered from the former. The design and simulation results with the SPPC parameters show the convincing effectiveness of the method. Two major features are (i) just downstream of the transverse collimation section, the protective collimators at the SC dipoles can effectively clean the particles with a large momentum deviation produced in the transverse collimators, with the downstream multistage momentum collimation section intercepting any further leakage, thus practically eliminating the beam loss in the downstream DS section; and (ii) the application of SC quadrupoles in the transverse collimation section can help create one more collimation stage which turns out to be very effective in reducing beam loss in the momentum collimation and experimental sections. Simulations with the FLUKA code have proven that, with some protection design, the SC magnets in the collimation section can be safe from quenches caused by the radiation effect. The main design goal of collimation inefficiency $3.55 \times 10^{-7} \mathrm{~m}^{-1}$ at the cold regions can be fulfilled very well. Although the details have been carried with the SPPC parameters, the method should be more general for proton colliders of such scale.

Eliminating the great risk of particle loss in the cold region due to cleaning beam halo particles, for the colliders with ultrahigh luminosity, it is foreseen that collision debris gives a significant contribution of particle losses around the experimental points. With the great challenges to the optical design and protection scheme of experimental insertions, it may be effective to apply the momentum collimation method in the same long straight sections to avoid the cold losses in the downstream DSs of the experimental regions. This work should be done in the future.

\section{ACKNOWLEDGMENTS}

The authors thank James Molson of CERN and Jianshu Hong of IHEP for supporting the MERLIN code at IHEP and also the SPPC colleagues for discussions. This work was supported by the National Natural Science Foundation of China (Projects No. 11575214, No. 11527811, and No. 11235012).

\section{APPENDIX: BRIEF OF THE DESIGN FEATURES AND MAIN PARAMETERS OF THE SPPC}

The SPPC is a next-generation proton-proton collider aiming for energy-frontier physics, especially for beyond the standard model research. It is the second phase of the CEPC-SPPC project which was proposed by Chinese scientists, and the two colliders use the same tunnel. As a future proton-proton collider, the SPPC will collide protons at a center of mass energy of $75 \mathrm{TeV}$, with a circumference of $100 \mathrm{~km}$ and a nominal luminosity of $1.01 \times 10^{35} \mathrm{~cm}^{-2} \mathrm{~s}^{-1}$ per IP. There is also an upgrading plan with higher energy (125-150 TeV). In the baseline

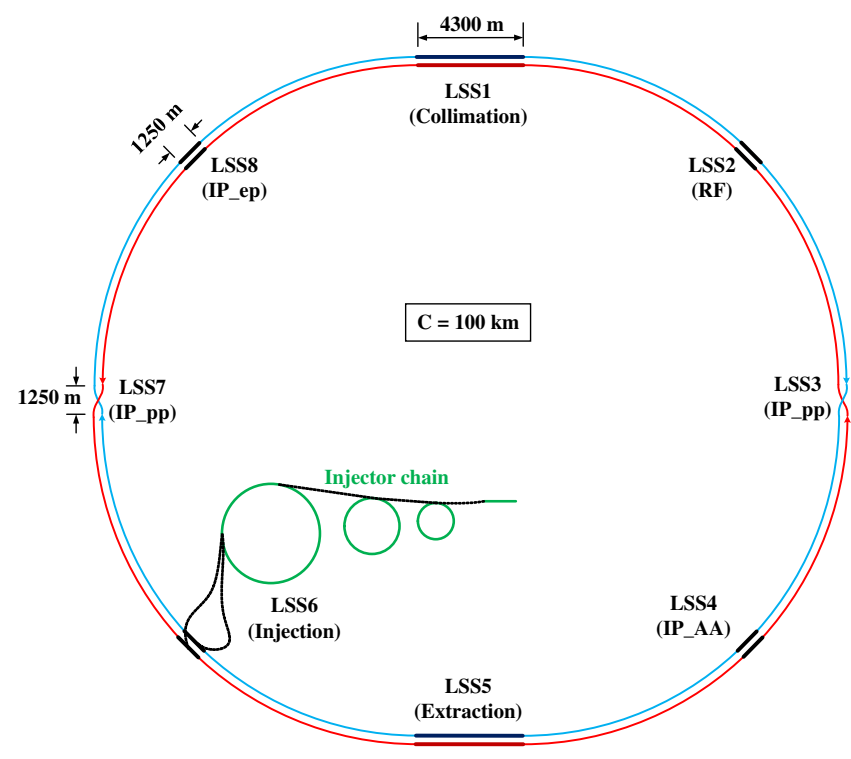

FIG. 18. The layout of the SPPC.

design, full iron-based HTS technology will be used in the SC magnets, and the field strength of the main dipoles is 12 T. Figure 18 shows the layout of the SPPC, and Table IV shows the main parameters given by the CEPC Conceptual Design Report (CDR) [39].

The SPPC is a complex accelerator facility and will be able to support research in different fields of physics, similar to the multiuse accelerator complex at CERN. Besides the energy-frontier physics program in the collider, the beams from each of the four accelerators in the injector chain can also support their own physics programs. The four stages, shown in Fig. 19, are a proton linac (p-Linac), a rapid cycling synchrotron (p-RCS), a medium-stage synchrotron (MSS), and the final stage synchrotron (SS). This research can occur during periods when a beam is not required by the next-stage accelerator.

TABLE IV. Main parameters of the SPPC.

\begin{tabular}{lcc}
\hline \hline Parameter & Unit & CDR \\
\hline Circumference & $\mathrm{km}$ & 100 \\
c.m. energy & $\mathrm{TeV}$ & 75 \\
Dipole field & $\mathrm{T}$ & 12 \\
Injection energy & $\mathrm{TeV}$ & 2.1 \\
Number of IPs & $\cdots$ & 2 \\
Nominal luminosity per IP & $\mathrm{cm}^{-2} \mathrm{~s}^{-1}$ & $1.0 \times 10^{35}$ \\
Beta function at collision & $\mathrm{m}$ & 0.75 \\
Circulating beam current & $\mathrm{A}$ & 0.7 \\
Bunch separation & $\mathrm{ns}$ & 25 \\
Bunch population & $\ldots$ & $1.5 \times 10^{11}$ \\
Number of bunches & $\ldots$ & 10080 \\
Normalized emittance & $\mu \mathrm{m}$ & 2.4 \\
SR power per beam & $\mathrm{MW}$ & 1.1 \\
SR heat load per aperture @arc & $\mathrm{W} / \mathrm{m}$ & 13 \\
\hline \hline
\end{tabular}


[1] R. Assmann et al., Equilibrium beam distribution and halo in the LHC, CERN-LHC-Project Reports No. 592, 2002.

[2] L. Evans and P. Bryant, LHC machine, J. Instrum. 3, S08001 (2008).

[3] R. W. Assmann, Collimators and beam absorbers for cleaning and machine protection, LHC Project Workshop, Chamonix XIV (CERN, Geneva, 2005), p. 261.

[4] G. Robert-Demolaize, Design and performance optimization of the LHC collimation system, Ph.D. thesis, Universite Joseph Fourier, Grenoble, 2006.

[5] R. W. Assmann et al., The final collimation system for the LHC, Proceedings of the 10th European Particle Accelerator Conference, Edinburgh, Scotland, 2006 (EPS-AG, Edinburgh, Scotland, 2006), p. 986.

[6] R. W. Assmann et al., Tests of tight collimator settings in the LHC, Report No. CERN-ATS-Note-2012-022 MD, 2012.

[7] B. Salvachua et al., Cleaning performance of the LHC collimation system up to $4 \mathrm{TeV}$, Proceedings of the 4th International Particle Accelerator Conference, IPAC2013, Shanghai, China, 2013 (JACoW, Shanghai, China, 2013), p. 1002.

[8] G. Apollinari et al., High-Luminosity Large Hadron Collider (HL-LHC) preliminary design report: Chap. 5, Report No. CERN-2015-005, 2015.

[9] D. Mirarchi et al., Cleaning performance of the collimation system of the High Luminosity Large Hadron Collider, in Proceedings of the 7th International Particle Accelerator Conference, Busan, Korea, 2016 (JACoW, Geneva, 2016), pp. 2494-2497, WEPMW030, http://doi.org/10.18429/ JACoWIPAC2016-WEPMW030.

[10] R. Bruce, C. Bracco, R. De Maria, M. Giovannozzi, A. Mereghetti, D. Mirarchi, S. Redaelli, E. Quaranta, and B. Salvachua, Reaching record-low beta* at the CERN Large Hadron Collider using a novel scheme of collimator settings and optics, Nucl. Instrum. Methods Phys. Res., Sect. A 848, 19 (2017).

[11] A. Marsiliy et al., Simulations of collimation cleaning performance with HL-LHC optics, Proceedings of the 4th International Particle Accelerator Conference, IPAC2013, Shanghai, China, 2013 (JACoW, Shanghai, China, 2013), MOPWO042.

[12] S. Redaelli, Collimation upgrades for HL-LHC, LHC Performance Workshop, Chamonix, France, 2014 (2014), https://indico.cern.ch/event/448109/contributions/ 1942040/attachments/1218992/1781341/SRedaelli_201601-28_print.pdf.

[13] T. Weiler et al., Beam cleaning and beam loss control, Proceedings of Hadron Beam 2008, Nashville, TN, USA, 2008 (2008), pp. 359-362, WGD08.

[14] R. Bruce et al., Cleaning performance with 11T dipoles and local dispersion suppressor collimation at the LHC, in Proceedings of the 5th International Particle Accelerator Conference, Dresden Germany, 2014 (JACoW, Geneva, 2014), pp. 170-173, CERN-ACC-2014-0129, MOPRO042, http://doi.org/10.18429/JACoW-IPAC2014-MOPRO042.

[15] K. Goulianos, Diffractive interactions of hadrons at high energies, Phys. Rep. 101, 169 (1983).

[16] K. Goulianos and J. Montanha, Factorization and scaling in hadronic diffraction, Phys. Rev. D 59, 114017 (1999).
[17] R. W. Assmann, Concept of a collimation system with enhanced operational stability and performance, FCC Week 2015, Washington, USA, 2015 (2015), https://indico .cern.ch/event/340703/contributions/802099/attachments/ 668687/919119/2015-03-25_FCC_Assmann-out.pdf.

[18] R. B. Appleby, R. J. Barlow, J. G. Molson, M. Serluca, and A. Toader, The practical Pomeron for high energy proton collimation, Eur. Phys. J. C 76, 520 (2016).

[19] M. Fiascaris, R. Bruce, and S. Redaelli, A conceptual solution for a beam halo collimation system for the Future Circular hadron-hadron Collider (FCC-hh), Nucl. Instrum. Methods Phys. Res., Sect. A 894, 96 (2018).

[20] D. I. Kaltchev et al., Optics solutions for the collimation insertions of LHC, Proceedings of the 18th Particle Accelerator Conference, New York, 1999, edited by A. Luccio and W. MacKay (IEEE, New York, 1999), p. 2623.

[21] J. Molson et al., Simulating the LHC collimation system with the accelerator physics library MERLIN, and loss map results, Proceedings of the 3rd International Particle Accelerator Conference, New Orleans, LA, 2012 (IEEE, Piscataway, NJ, 2012), pp. 12-14.

[22] M. Serluca et al., HL-LHC collimation studies with MERLIN code, in Proceedings of the 5th International Particle Accelerator Conference IPAC 2014, Dresden, Germany, 2014 (JACoW, Geneva, Switzerland, 2014), pp. 784-786, MOPRI077.

[23] R. B. Appleby et al., Merlin source code Web site, https:// github.com/MERLIN-Collaboration/MERLIN.

[24] The CEPC-SPPC Study Group, CEPC-SPPC Preliminary Conceptual Design Report, Vol. II: Accelerator, 2015, http://cepc.ihep.ac.cn/preCDR/main_preCDR.pdf.

[25] M. I. Besana et al., Energy deposition in the betatron collimation insertion of the $100 \mathrm{TeV}$ future circular collider, in Proceedings of 8th International Particle Accelerator Conference IPAC 2017, Copenhagen, Denmark, 2017 (JACoW, Geneva, Switzerland, 2017), p. 68, MOPAB003.

[26] M. Varasteh, Energy deposition in the FCC-hh betatron cleaning insertion, FCC Week 2018, Amsterdam, Netherlands, 2018, (2018), https://indico.cern.ch/event/656491/ contributions/2930758/attachments/1629679/2597131/ Varasteh_BeamLossInCollimators_2018-04-10.pdf.

[27] N. Mounet et al., Collimator impedance, LHC collimation review, CERN, Geneva, 2013, https://indico.cern.ch/event/ 251588/session/1/contribution/6/material/slides/0.pdf.

[28] T. Trenkler and J. B. Jeanneret, The principles of two stage betatron and momentum collimation in circular accelerators, Part. Accel. 50, 287 (1995).

[29] D. I. Kaltchev et al., Numerical optimization of collimator jaw orientations and locations in the LHC, Proceedings of the Particle Accelerator Conference, Vancouver, BC, Canada, 1997 (IEEE, New York, 1997), p. 153.

[30] D. Kaltchev et al., Optimization of collimator jaw locations for the LHC, Proceedings of the EPAC96 Conference, Barcelona, 1996, edited by S. Myers et al. (1996), p. 1432.

[31] J. B. Jeanneret, Optics of a two-stage collimation system, Phys. Rev. ST Accel. Beams 1, 081001 (1998).

[32] O. Bruning et al., LHC Design Report No. CERN-2004003 Vol. I: Chap. 4, 2004.

[33] R. Assmann et al., Integration of tertiary collimators, beam-beam rate monitors and space reservation for a 
calorimeter in the experimental LSS's, CERN LHC Project Document No. LJ-EC-0003, 2004.

[34] O. Bruning et al., LHC Design Report No. CERN-2004003, Vol. I: Chap. 18, 2004.

[35] R. Bruce et al., Sources of machine-induced background in the ATLAS and CMS detectors at the CERN Large Hadron Collider, Nucl. Instrum. Methods Phys. Res., Sect. A 729, 825 (2013).

[36] ATLAS Collaboration, Beam-induced and cosmic-ray backgrounds observed in the ATLAS detector during the LHC 2012 proton-proton running period, J. Instrum. 11 (2016) P05013.

[37] R. Assmann et al., Requirements for the LHC collimation system, Proceedings of the 8th European Particle Accelerator Conference, Paris, 2002 (EPS-IGA and CERN, Geneva, 2002), p. 197.

[38] J. B. Jeanneret et al., Quench levels and transient beam losses in LHC magnets, CERN-LHC-Project Report No. 44, 1996.

[39] The CEPC Study Group, CEPC Conceptual Design Report, Vol. I: Accelerator, 2018, http://cepc.ihep.ac.cn/CDR_v6_ 201808.pdf.

[40] Q. Xu et al., 20-T dipole magnet with common-coil configuration: Main characteristics and challenges, IEEE Trans. Appl. Supercond. 26, 4000404 (2016).

[41] C. Bracco, Commissioning scenarios and tests for the LHC collimation system, Ph.D. thesis, EPFL Lausanne, 2008.

[42] D. Schulte, FCC-hh machine layout and optics, FCC Week 2016, Rome, Italy, 2016 (2016), https://indico.cern.ch/event/ 438866/contributions/1085167.

[43] J.G. Molson, Proton scattering and collimation for the LHC and LHC luminosity upgrade, Ph.D. Thesis, University of Manchester, 2014.

[44] M. Serluca et al., Comparison MERLIN/SixTrack for LHC collimation studies, in Proceedings of the 5th International Particle Accelerator Conference, IPAC 2014, Dresden, Germany, 2014 (JACoW, Geneva, Switzerland, 2014), pp. 185-187, MOPRO046.
[45] J. Tang et al., Concept for a future super proton-proton collider, arXiv:1507.03224.

[46] R. Bruce et al., Simulations and measurements of beam loss patterns at the CERN Large Hadron Collider, Phys. Rev. ST Accel. Beams 17, 081004 (2014).

[47] G. Robert-Demolaize et al., A new version of SixTrack with collimation and aperture interface, Proceedings of the 21st Particle Accelerator Conference, Knoxville, TN, 2005 (IEEE, Piscataway, NJ, 2005), p. 4084.

[48] D. Bocian, B. Dehning, and A. Siemko, Modeling of quench limit for steady state heat deposits in LHC magnets, IEEE Trans. Appl. Supercond. 18, 112 (2008).

[49] B. Auchmann et al., Testing beam-induced quench levels of LHC SC magnets, Phys. Rev. ST Accel. Beams 18, 061002 (2015).

[50] P. P. Granieri and R. van Weelderen, Deduction of steady state cable quench limits for various electrical insulation schemes with application to LHC and HL-LHC magnets, IEEE Trans. Appl. Supercond. 24, 4802806 (2014).

[51] E. Todesco, Quench limits in the next generation of magnets, arXiv:1401.3931.

[52] T. T. Böhlen, F. Cerutti, M. P. W. Chin, A. Fassò, A. Ferrari, P. G. Ortega, A. Mairani, P. R. Sala, G. Smirnov, and V. Vlachoudis, The FLUKA code: Developments and challenges for high energy and medical applications, Nucl. Data Sheets 120, 211 (2014).

[53] G. Battistoni, F. Cerutti, A. Fassò, A. Ferrari, S. Muraro, J. Ranft, S. Roesler, and P. R. Sala, The FLUKA code: Description and benchmarking, AIP Conf. Proc. 896, 31 (2007). We used FLUKA2008, which was the latest version at the time of this study.

[54] Y. Ma, Progress in wire fabrication of iron-based superconductors, Supercond. Sci. Technol. 25, 113001 (2012).

[55] H. Rafique et al., Proton cross-talk and losses in the dispersion suppressor regions at the FCC-hh, in Proceedings of the 8th International Particle Accelerator Conference, IPAC 2017, Copenhagen, Denmark, 2017 (JACoW, Geneva, Switzerland, 2017), pp. 1763-1765, TUPIK037. 